\title{
No Role for Activated Long-term Memory in Attentional Control Settings
}

\author{
by \\ Lindsay Amber Plater
}

\author{
A Thesis \\ presented to \\ The University of Guelph
}

In partial fulfilment of requirements for the degree of

Master of Science

in

Psychology and Neuroscience

Guelph, Ontario, Canada

(C) Lindsay Plater, August, 2018 


\section{ABSTRACT \\ NO ROLE FOR ACTIVATED LONG-TERM MEMORY IN}

\section{ATTENTIONAL CONTROL SETTINGS}

Lindsay Amber Plater

University of Guelph, 2018
Advisor:

Dr. Naseem Al-Aidroos

Attentional control settings (ACSs) allow us to constrain which objects in our complex visual environment capture attention. Recent research has shown that ACSs can be maintained by long-term memory (LTM); but what is special about LTM ACSs that they bias attentional capture, when other LTMs do not? Is it possible that they have been activated? To test this, we mixed spatial blink trials to test for the presence of an ACS, and working memory trials to test for representation in activated LTM (ALTM). We induced participants to adopt an LTM ACS for 30 (Experiment 1) or 15 (Experiment 2) complex visual objects, but this did not consistently result in representation in ALTM for those objects. We induced participants to represent objects in ALTM (Experiments 3 and 4), but this did not result in an ACS for those objects. We conclude that LTM ACSs are not accomplished through ALTM. 


\section{DEDICATION}

This thesis is dedicated to my partner, Aaron. Thank you for holding me when the days seemed desolate; you are my dragon. 


\section{ACKNOWLEDGEMENTS}

This list of acknowledgements has to start with my advisor, Dr. Naseem Al-Aidroos. Your guidance, encouragement, and thoughtful conversations have helped shape both this thesis and my research abilities to what they are today. Here's to our first of many publications together...once it gets accepted, of course! Also, your dedication to making sure I graduate on time has been so greatly appreciated; thanks for staying up long into the night—even on weekends! — passing the manuscript back and forth. It made a world of difference.

I would also like to extend warm thanks to Dr. Chris Fiacconi, my M.Sc. advisory committee member as well as my practicum supervisor. Your unbridled enthusiasm is sure to be the source of many productive projects together.

Special thanks to Drs. Maria Giammarco and Naseem Al-Aidroos for providing me with the spatial blink code and the working memory code to get me started in Fall 2016.

Thanks has to be given to the other members of the Visual Cognitive Neuroscience lab, as well as to the Cognitive-Affective Lab up the hall. Lab meetings have been a great source of camaraderie, while also providing an opportunity to develop my communication skills. Thanks for listening and offering your insights!

Also, a shout-out to Robin Sorbara, Graduate Program Assistant for the Department of Psychology. You're like the helpful aunt who has all the answers to my questions; your timely advice helped to prevent more than one mental breakdown.

No acknowledgement section would be complete without thanking my friends and family. While you may not really understand what exactly it is that I do, what matters is that you have supported me along the way, no matter what. I love you all.

Lastly, this research was made possible thanks to funding from the provincial and the federal governments; thank you. 


\section{TABLE OF CONTENTS}

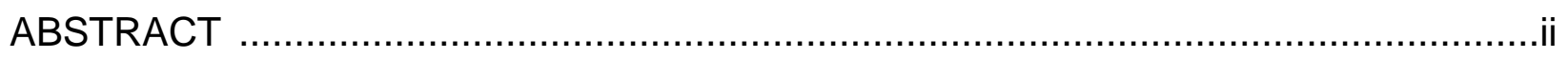

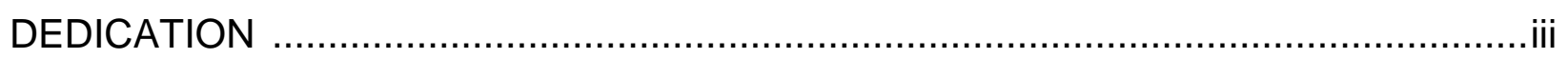

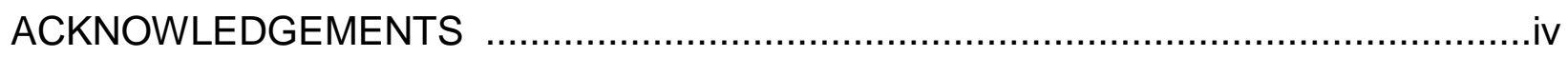

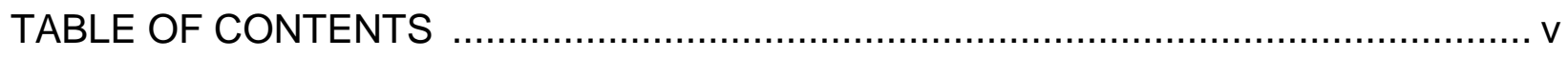

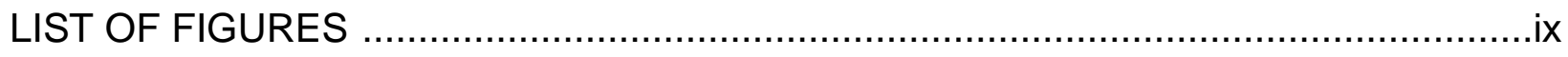

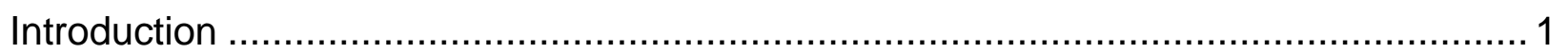

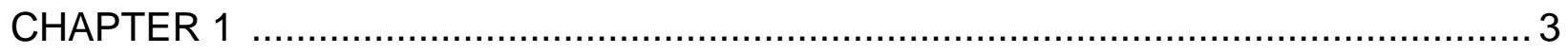

Visual LTM and Visual Working Memory Both Contribute to ACSs .......................... 3

The Embedded-processes Model of Memory ……............................................... 6

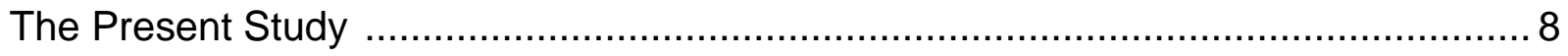

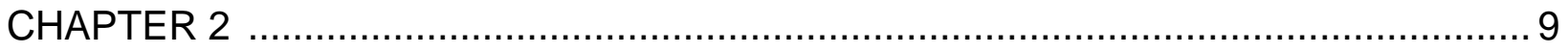

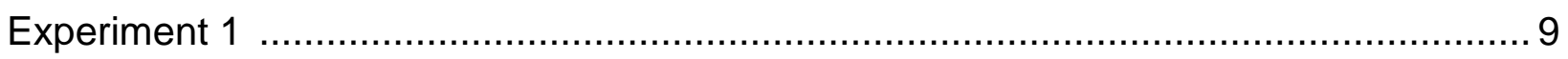

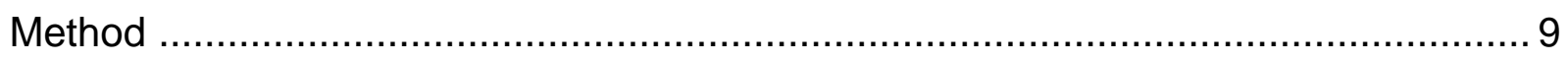

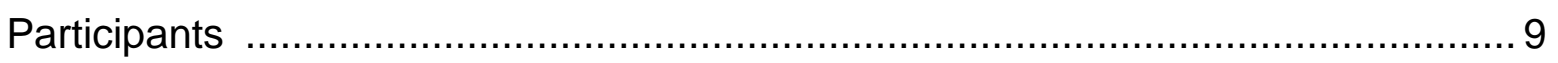

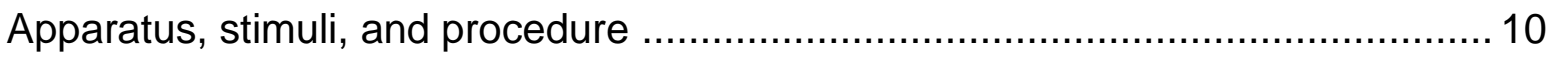

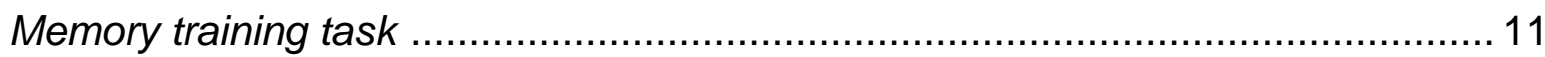

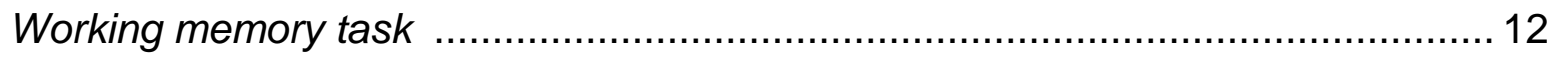

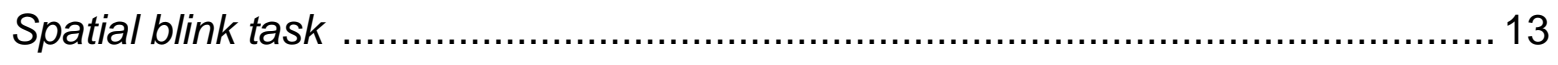

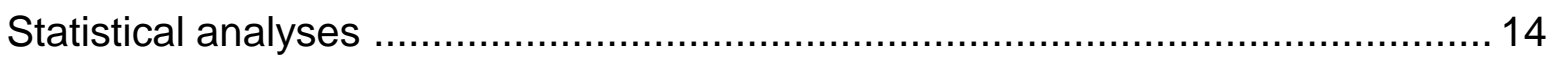

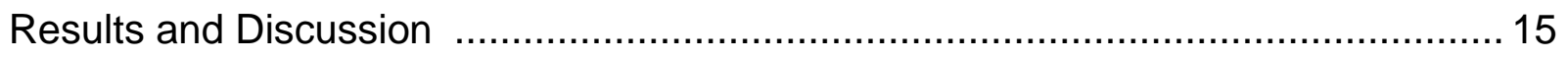




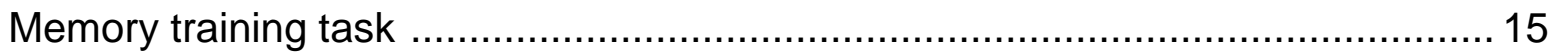

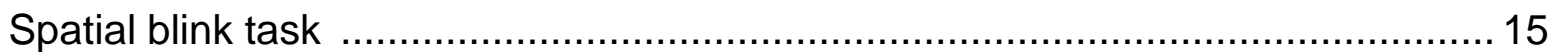

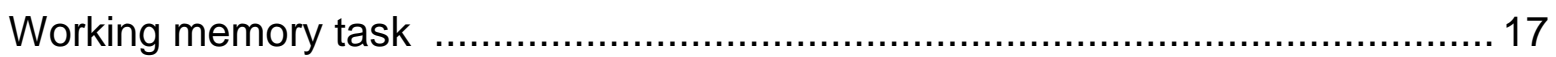

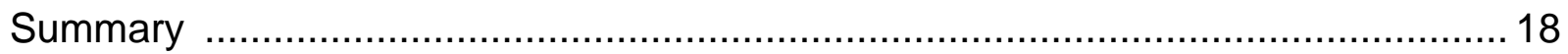

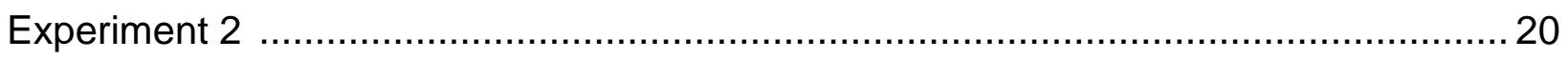

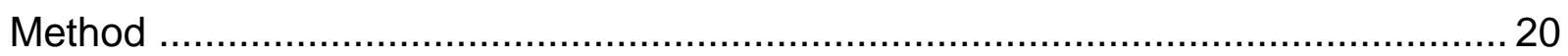

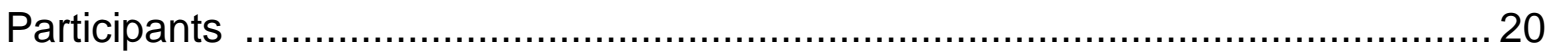

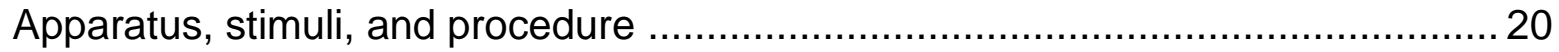

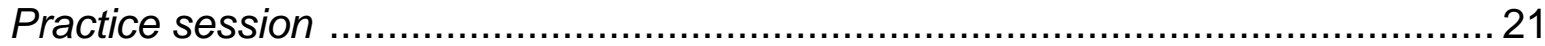

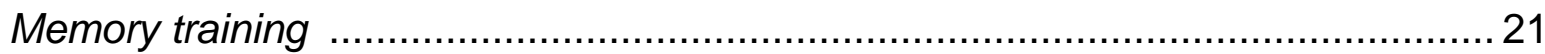

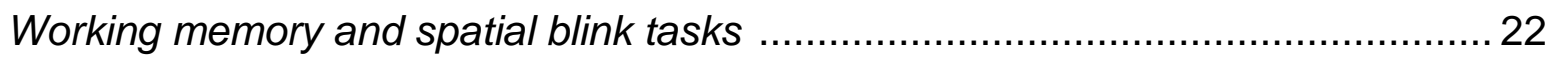

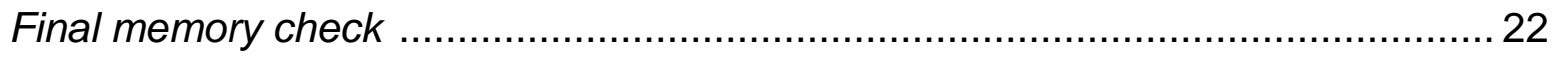

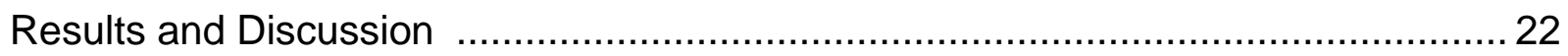

Memory training and final memory check ..................................................... 22

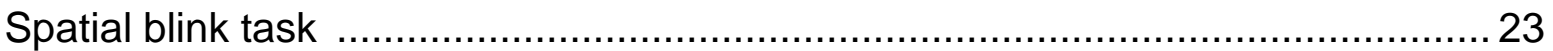

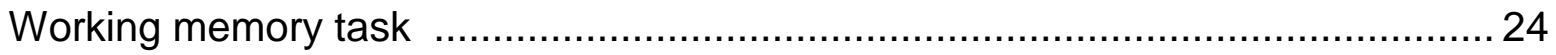

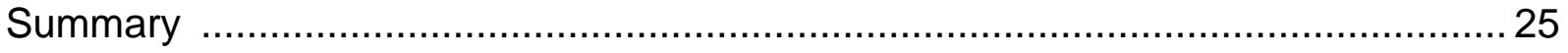

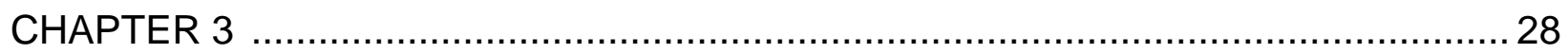

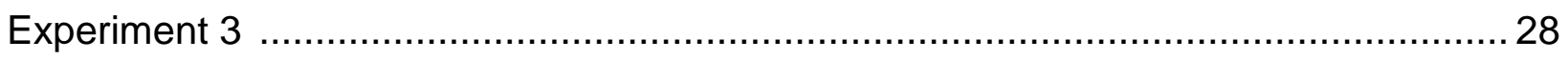

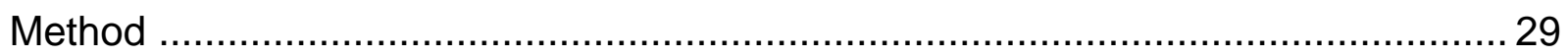

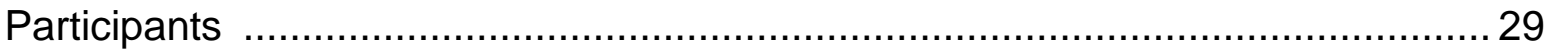

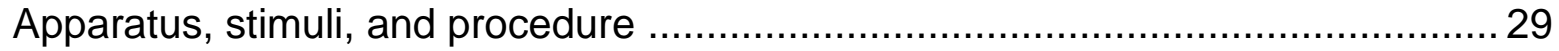

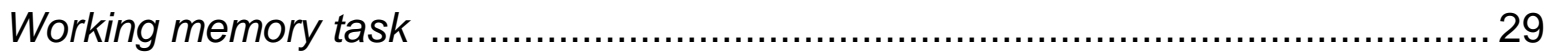




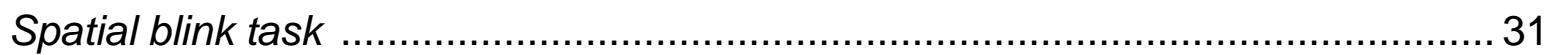

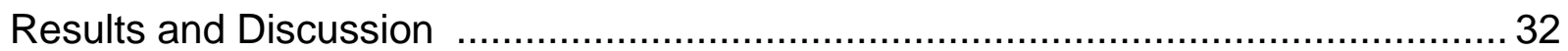

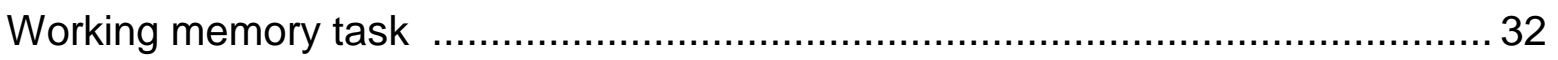

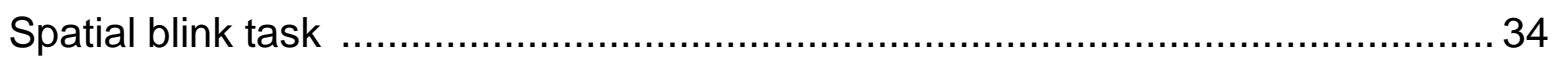

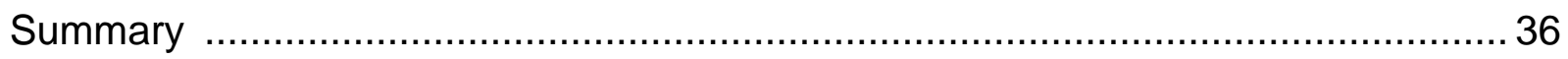

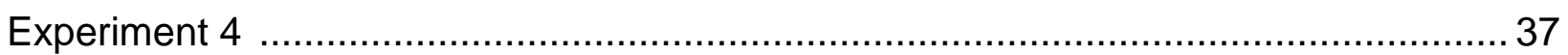

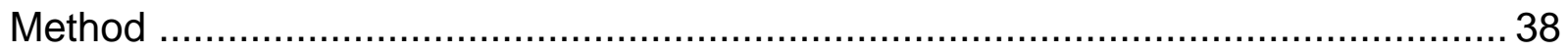

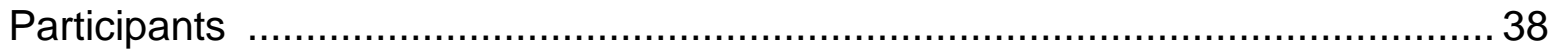

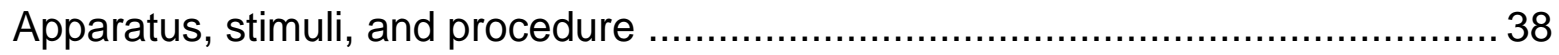

Working memory and spatial blink tasks ....................................................... 38

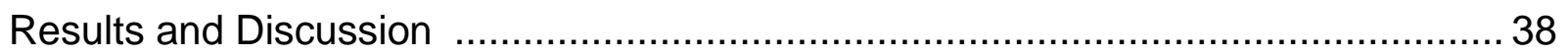

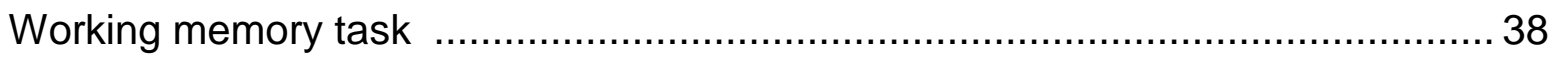

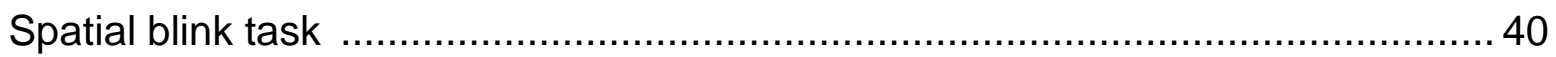

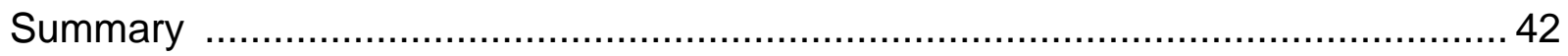

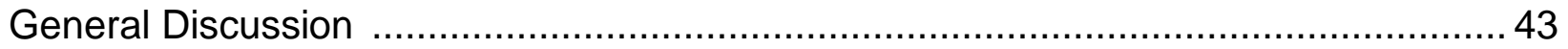

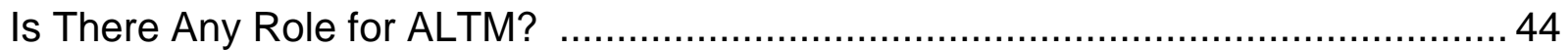

Attentional Control Settings vs. Attentional Templates ......................................... 45

What Distinguishes ACS Representations in LTM, if not ALTM? ........................... 46

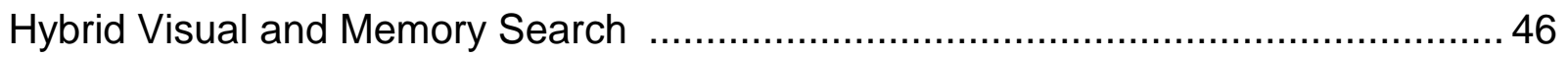

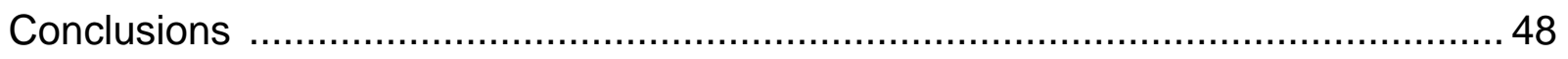

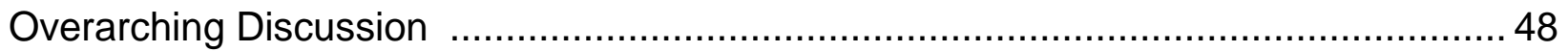

Limitations and Future Directions ............................................................... 48 


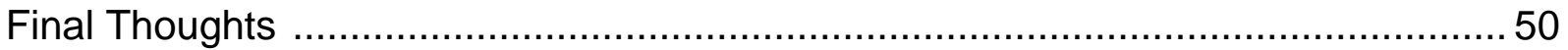

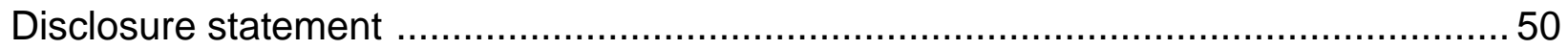

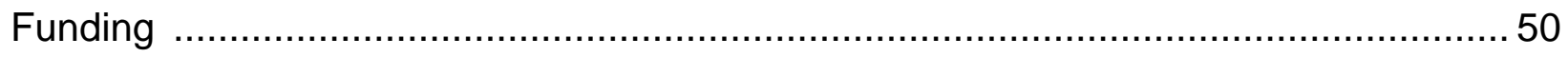

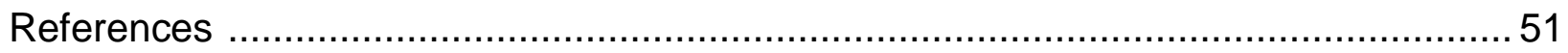




\section{LIST OF FIGURES}

Figure 1. Sample memory training task for Experiment 1. A) Study phase.

B) Recognition test phase

Figure 2. Sample trial sequences for Experiments 1 and 2. A) Trial sequence for the working memory task. B) Trial sequence for the spatial blink task 13

Figure 3. Accuracy data for the spatial blink (SB) task for Experiment 1 ................... 16

Figure 4. Reaction time data for the working memory task for Experiment $1 \quad \ldots \ldots \ldots \ldots . . .17$

Figure 5. Correlation between attentional capture and the intrusion effect for Experiment 1

Figure 6. Accuracy data for the spatial blink (SB) task for Experiment 2 ................... 23

Figure 7. Reaction time data for the working memory task for Experiment $2 \quad$.............. 24

Figure 8. Correlation between attentional capture and the intrusion effect for Experiment 2

Figure 9. Sample trial sequences for Experiments 3 and 4. A) Trial sequence for the working memory task. B) Trial sequence for the spatial blink task

Figure 10. Set size reaction time data for Experiment 3's working memory task 33

Figure 11. Reaction time data for two probe types for Experiment 3's working memory task

Figure 12. Accuracy data for the spatial blink (SB) task for Experiment 3 35

Figure 13. Correlation between attentional capture and the intrusion effect for Experiment 3

Figure 14. Set size reaction time data for Experiment 4's working memory task

Figure 15. Reaction time data for two probe types for Experiment 4's working memory task

Figure 16. Accuracy data for the spatial blink (SB) task for Experiment 4 41

Figure 17. Correlation between attentional capture and the intrusion effect for Experiment 4 


\section{Introduction}

Chapters 1, 2, and 3 have been submitted for publication. The manuscript was divided into chapters for the present thesis, but otherwise the chapters are unchanged from the main text of the submitted version.

Plater, L, Giammarco, M., Fiacconi, C., \& Al-Aidroos, N. (submitted). No role for activated long-term memory in attentional control settings. Journal of Experimental Psychology: General.

Given that our complex visual environments contain far more information than we could possibly attend to at once, how are we able to attend to certain stimuli, while ignoring others? If you are walking down a crowded street looking for your friend, and your friend has told you that they are wearing their favourite red shirt today, based on your goal of "look for red", stimuli that are red will capture your attention, however, stimuli that are not red will not (Folk \& Remington, 1998). This ability is known to researchers as an attentional control setting (ACS). Briefly, an ACS allows you to constrain what objects in the environment will capture your attention based on your current goals.

While ACSs have been researched for several decades, a recent topic of research has been trying to determine which memory system supports ACSs. While much of the previous research has demonstrated that working memory is capable of maintaining ACSs, work in our laboratory has demonstrated that ACSs can also be accomplished through episodic long-term memory (LTM; Giammarco, Paoletti, Guild, \& Al-Aidroos, 2016). But this work still leaves an open question: Given that LTM contains a lifetime of memories, and given that the vast majority of these memories have no direct effect on attention, what is special about LTM ACSs that allow some representations in LTM to bias the capture of attention, when others do not? This thesis deals with one potential answer to this question; that some LTM representations have been activated, and are able to interface with perception to bias attentional capture. 
In Chapter 1 of this thesis, I present relevant background research on ACSs, and also provide a short overview of the methodology used in Chapters 2 and 3. In Chapter 2, I describe two experiments that induced participants to adopt an ACS for multiple complex visual objects, and then tested whether those same objects were represented in activated LTM (ALTM). To preview the results, while participants successfully adopted an ACS for the objects, those same objects were not consistently represented in ALTM. In Chapter 3, I describe two experiments where we used the same techniques to investigate the opposite account. For this chapter, we induced participants to represent complex visual objects in ALTM, and then tested whether that was sufficient for those same objects to form an ACS and bias attentional capture. Again, to preview the results, while participants successfully represented the objects in ALTM, those same objects did not form an ACS. The thesis ends with a final Discussion section that reiterates the main findings from this research, extends this research to a similar field (hybrid visual and memory search), discusses some of the limitations of the current work, and proposes some future directions. 


\section{Chapter 1: Background on Attentional Control Settings}

While it may sometimes feel like our attention is automatically drawn to salient sensory visual events, such as the flashing lights on a passing ambulance, the ability for such events to capture our attention is under our control. When we actively search our environment for one type of visual information (e.g., a friend wearing a red baseball cap), we adopt an attentional control setting (ACS) for the property that defines our target (i.e., red), which ensures that only ACS-matching stimuli possessing that property will capture our attention (Folk, Remington, \& Johnston, 1992; Folk, Remington, \& Wright, 1994). Formally, attentional capture is said to be contingent on ACSs; red stimuli will capture our attention when we are looking for something red, but not when we are looking for something green (Folk \& Remington, 1998).

At present, we do not have a full understanding of how ACSs control capture. One aspect of this control process that is under active investigation is the memory system that stores the identity of the searched-for target(s) (Berggren \& Eimer, 2018; Giammarco et al., 2016; Goodhew, Kendall, Ferber, \& Pratt, 2014; Olivers, Peters, Houtkamp, \& Roelfsema, 2011; Woodman, Carlisle, \& Reinhart, 2013; Wyble, Folk, \& Potter, 2013). Interestingly, episodic LTM is one of the memory systems that has been shown to maintain the contents of ACSs; we can simultaneously search our environment for at least 30 different visual objects by adopting an ACS based on representations of those objects in LTM, with the result that only those 30 objects will capture attention (Giammarco et al., 2016). But this finding also raises a question: Given the vast amount of information represented in LTM, what is special about the LTM representations that comprise an ACS, such that these representations bias attentional capture when other LTM representations do not?

\section{Visual LTM and Visual Working Memory Both Contribute to ACSs}

The most direct evidence that ACSs can be established based on representations in LTM comes from a recent study by our research group (Giammarco et al., 2016; see 
also Carlisle, Arita, Pardo, \& Woodman, 2011, for related work on attentional templates in LTM). Attentional control settings are typically studied by measuring attentional capture using a Posner cueing task (Posner \& Cohen, 1984) with task-irrelevant attentional cues. The conventional finding is that, when participants are monitoring the environment for the appearance of a particular visual target, only pre-cues that possess the target defining property (e.g., are the same colour as the target) will capture attention and produce a cueing effect (i.e., faster target response times when the target appears at the cued location than elsewhere; Folk \& Remington, 1998; Folk, Remington, \& Johnston, 1992; Folk, Remington, \& Wright, 1994). To assess the possibility that ACSs can be defined based on representations in LTM, Giammarco et al. (2016) had participants memorize a list of 30 everyday, complex visual objects-a feat accomplished through LTM (Brady, Konkle, Alvarez, \& Oliva, 2008; Guild, Cripps, Anderson, \& Al-Aidroos, 2014; Wolfe, 2012)—and then used these items as the targets in a Posner cueing task. They found that the only cue stimuli to produce attentional capture were the objects from the memorized list, suggesting that participants had adopted an ACS for these LTM representations.

To provide converging evidence for their findings, Giammarco et al. (2016) measured attentional capture using another task commonly used to study ACSs: the spatial blink task (Folk, Leber, \& Egeth, 2002). We also used this type of task in the present study. Giammarco et al. (Experiment 3, 2016) had participants memorize two separate lists that each contained 15 everyday visual objects ("List A" and "List B"), and then designated one of the lists as the targets for the spatial blink task. They used two lists in this experiment to show that ACSs can be constrained to a particular memory source (recollective memory), rather than being defined based on all recently memorized/familiar objects (Yonelinas, 2002). For the spatial blink task, participants monitored a centrally presented flashing stream of objects for the appearance of any one of the 15 target objects, and indicated at the end of the stream which one they had seen. Notably, a pair of spatial distractors was presented before the target appeared, with one distractor object above and one below the central stream. In such spatial blink 
tasks, if either of the distractors are part of an ACS, these distractors will capture attention, and detection of targets appearing shortly thereafter is impaired (Folk et al., 2002). Giammarco et al. (2016) found that the only stimuli that captured attention were the 15 target objects; novel objects and, critically, the equally familiar 15 memorized non-target objects had no effect on participants' ability to report the target. Based on these findings, the authors concluded that ACSs can be defined based on episodic LTM representations. Yet our question remains: What is special about the LTM representations of the 15 target objects that allows capture to be constrained to only those objects, and not other objects represented in LTM?

While Giammarco et al.'s (2016) study demonstrates a role for LTM in establishing ACSs, it is not the only memory system that is capable of doing so. Indeed, the majority of past research examining memory and ACSs has focussed on visual working memory, an online memory system that is limited to storing accurate representations of about three to four visual objects (Luck \& Vogel, 1997; Phillips, 1974). It may be possible to derive insights into the mechanisms underlying LTM-based ACSs from this visual working memory research. Much of the evidence that ACSs are supported by visual working memory comes from the demonstration that having participants remember a particular object using visual working memory (e.g., a red square) often causes attention to be drawn to similar stimuli in the environment (i.e., other red stimuli; Olivers, Meijer, \& Theeuwes, 2006; Soto, Heinke, Humphreys, \& Blanco, 2005). However, not all representations in visual working memory will necessarily bias attentional capture; for example, when participants are asked to remember two colours, it is often the case that at most one of these representations will bias attention (Olivers, 2009; although see Beck, Hollingworth, \& Luck, 2012). As an account of this observation, Olivers, Peters, Houtkamp, and Roelfsema (2011) proposed that capture is determined by the state of representation in visual working memory: one representation can be in an active state, forming an attentional template that biases attention, and any other representations are in an accessory state that do not interact with attention. Accordingly, it appears that 
observers can adopt an ACS for, say, the colour red, by representing a red stimulus in visual working memory in the active state.

Perhaps a similar distinction between states of representation also determines which LTM representations bias attention. Might it be that the objects that comprise an LTM ACS capture attention when encountered in the environment because they are represented in an active state within LTM? Relevantly, the embedded-processes model of memory (Cowan, 1988; Oberauer, 2002) proposes a distinction between active and non-active LTM representations, and, beyond the similarity in nomenclature, aspects of this active LTM state make it well suited to supporting LTM ACSs.

\section{The Embedded-processes Model of Memory}

According to the embedded-processes model of memory, "memory" is a single, hierarchically organized store. The components of this model have changed slightly over time; that said, according to a recent version (Oberauer \& Hein, 2012), memory comprises: 1) LTM, a potentially limitless store; 2) active long-term memory (ALTM), the subset of LTM representations that were recently, or are currently, attended; 3) the region of direct access, a subset of ALTM representations that are currently within the broad focus of attention; and 4) the narrow focus of attention, a single representation within the region of direct access. Information represented within the region of direct access is consciously accessible; however, because attention is capacity limited, the amount of information stored in this region is constrained to about four items or chunks. Thus, the region of direct access is comparable to what we referred to above as working memory, with the narrow focus of attention being equivalent to the active state within 
working memory ${ }^{1}$. In contrast to attention, which is capacity limited, activation is time limited (Cowan, 1999); accordingly, the capacity of ALTM is larger than that of the region of direct access, and, indeed, has no known limit. Moreover, unlike other LTM representations, those in ALTM can directly interact with aspects of ongoing cognition, including recognition judgements of perceptual objects (Oberauer, 2001). Given the large potential capacity of ALTM, and the ability for ALTM representations to influence behaviour, this component of memory may be well suited to serve as the basis for LTM ACSs.

The main evidence that objects can be represented outside of working memory, yet still influence behaviour (i.e., be represented in ALTM), comes from Oberauer (2001), who measured intrusion effects with a modified Sternberg task. We also used a similar task in the present study. Oberauer (2001) had participants memorize and retain two short lists of words in working memory, and then tested memory at the end of the trial by presenting a single probe that required a recognition judgement. Prior to the probe, a cue was presented indicating that one list was no longer relevant, the idea being that participants may stop attending to these irrelevant items, leaving the items to only be represented in ALTM and not working memory. To assess whether irrelevant items were represented in working memory, Oberauer (2001) manipulated the set size of the irrelevant list (one vs. three words) and the time between the cue and memory probe. He found that response times (RTs) to memory probes that appeared within one second of the cue were longer for the larger irrelevant set size, suggesting that the irrelevant items were initially stored in working memory (Sternberg, 1966). After about one second, however, irrelevant list set size had no effect on probe RTs, suggesting they were no longer represented in working memory. While the number of irrelevant items ceased affecting probe RTs about one second after the cue, the identities of these items

\footnotetext{
${ }^{1}$ According to embedded-processes memory models, ALTM is also a component of working memory (Cowan, 1988; Oberauer, 2002), reflecting the fact that ALTM representations are sufficiently accessible to guide behaviour (i.e., do work). For the present paper, however, we refer to ALTM as a form of LTM to align with the typical characterization that working memory is limited to representing 3 to 4 chunks of information, and items stored beyond this limit are represented outside of working memory, in LTM (Carlisle et al., 2011; Luck \& Vogel, 1997; Olivers, 2009).
} 
continued to affect RTs for up to five seconds, the longest cue-probe interval used in the study. Specifically, on some trials an irrelevant item was presented as the memory probe, and participants took longer to correctly identify these probes as not being part of the relevant list than completely novel probes. This slowing of RTs is referred to as an intrusion effect, and demonstrates that ALTM representations outside of working memory can influence behaviour.

\section{The Present Study}

In the present study we investigated the potential role of ALTM in supporting LTM ACSs. Given the large capacity of ALTM, and given that ALTM representations can directly influence behaviour (i.e., without representation in working memory), we asked whether adopting an LTM ACS for a set of everyday visual objects is accomplished by representing those objects in ALTM. We henceforth refer to this possibility as the ALTM account of LTM ACSs. To investigate this account, across four experiments we combined a working memory task with a spatial blink task, allowing us to concurrently assess ALTM and ACSs, respectively, as well as their potential relationship. To preview the results, inducing participants to adopt an LTM ACS for 30 (Experiment 1) or 15 (Experiment 2) complex visual objects was not sufficient for those objects to be consistently represented in ALTM. Additionally, inducing participants to represent complex visual objects in ALTM (Experiments 3 and 4) was not sufficient to induce an ACS for those objects. These findings lead us to reject the ALTM account, and conclude that representation in ALTM is not the factor that determines which representations in LTM comprise an ACS, and which do not. 


\section{Chapter 2: Is Activated Long-term Memory Necessary for Adopting a Long-term}

\section{Memory Attentional Control Setting?}

\section{Experiment 1}

As a first step in investigating whether LTM ACSs are accomplished through ALTM, Experiment 1 tested the prediction that when participants adopt an ACS for complex visual objects, those objects should be represented in ALTM. Accordingly, like the original LTM ACS study by Giammarco et al. (2016), we had participants memorize 30 images of everyday objects and then search for those objects in a spatial blink task. This spatial blink task allowed us to both induce participants to adopt an ACS for the memorized objects, and measure contingent capture (Folk et al., 2002; Giammarco et al., 2016). New to this experiment, we also randomly intermixed spatial blink trials with trials from a working memory task, allowing us to also assess whether the memorized objects were represented in ALTM by measuring the intrusion effect (Oberauer, 2001). Interestingly, while the memorized/searched-for objects produced a small intrusion effect, closer examination of the results revealed that the magnitude of this effect was unrelated to a participant's ability to control attentional capture, providing preliminary evidence that LTM ACSs are not accomplished using ALTM, a conclusion corroborated by Experiments 2, 3, and 4 .

\section{Method}

Participants. For all studies, informed consent was obtained from each participant, experimental protocols were approved by the University of Guelph ethics board, and all participants reported having normal, or corrected-to-normal, visual acuity. Thirty-eight undergraduate students from the University of Guelph (mean age 18.1 years, 34 females) participated in Experiment 1 for partial course credit. Multiple participants had 
to be excluded from analyses due to the difficulty of the two tasks; this included two participants with error rates at $40 \%$ or higher on the working memory task, and 7 participants with error rates at $50 \%$ or higher on the spatial blink task. Thus, Experiment 1 had a final sample size of 29 participants. Notably, including all participants in the analyses reported below does not alter the pattern of results, except that the difference in RTs between ACS probes and nonACS probes in the working memory task becomes statistically non-significant, $t(35)=1.30, p=.101$, one-tailed, and the Bayesian pairedsamples $t$-test provides anecdotal evidence to support the null model, $\mathrm{BF}_{01}=1.45$, onetailed; this change is consistent with our conclusion that LTM ACSs are not accomplished through ALTM.

Apparatus, stimuli, and procedure. All experiments were conducted on a desktop computer with a $1280 \times 1024$ resolution $75 \mathrm{~Hz}$ CRT display, and responses were made on a standard keyboard. Participants used a head and chin rest to keep their gaze distance constant at $52 \mathrm{~cm}$ from the computer screen for the duration of the experiment.

For this experiment, participants first memorized 30 images of everyday objects, and then completed randomly intermixed trials of a spatial blink task and a working memory task. All object images were presented on a white background, and were unique objects selected from Brady, Konkle, Alvarez, and Oliva (2008); these were the same objects used in Giammarco et al. (2016). Objects were from many categories, including: food, animals, general household objects, tools, clothing, appliances, vehicles, and buildings. The 30 objects that participants memorized and then searched for in the spatial blink task - the ACS objects - were randomly selected without replacement for each participant from a pool of 125 objects. A second set of 30 objects-nonACS objectswas also randomly selected without replacement from this pool. In addition to being selected from the same pool of images, nonACS objects were shown equally as often as ACS objects during the working memory and spatial blink tasks, but they were never studied and were not targets for the spatial blink task. The nonACS objects were used to establish baseline measures of attentional capture and the intrusion effect. All other object images were randomly selected from a separate pool of 2,152 objects. 
Memory training task. The experiment began with a memory training task, see Figure 1. Participants memorized, and were tested on, the identities of the targets for the upcoming spatial blink task. Object images subtending $3.4 \times 3.4^{\circ}$ of visual angle appeared one at a time in the middle of a white screen for 3,000 ms each. The interstimulus interval lasted $1,000 \mathrm{~ms}$, and consisted of a white screen with a central fixation point that subtended $0.05^{\circ}$. After studying the objects, participants completed a two-item forced-choice recognition memory test for each of the 30 ACS objects. Participants were shown an ACS object and a randomly selected novel object, and were asked to indicate which one they had seen previously. To ensure that participants had adequately learned the identities of the ACS objects, participants completed the memory training task (both study and recognition test phases) until they achieved an accuracy of $80 \%$ or higher on the test phase twice consecutively (Giammarco et al., 2016; Wolfe, 2012). If participants responded incorrectly or did not make a response, a $500 \mathrm{~Hz}$ error tone played for $50 \mathrm{~ms}$ before the start of the next trial.

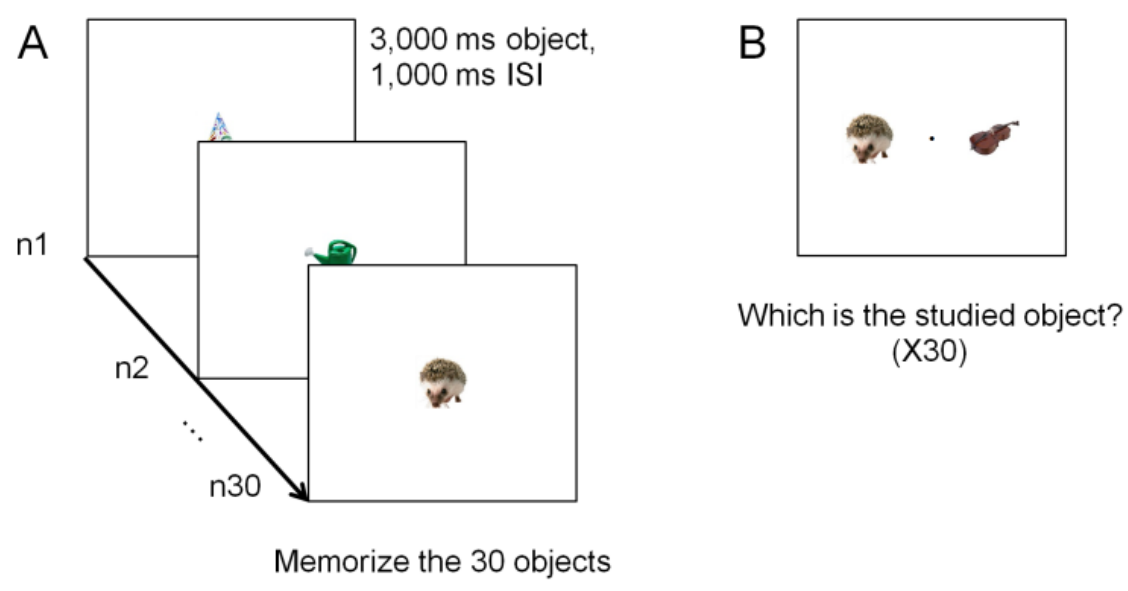

Figure 1. Sample memory training task for Experiment 1. A) Study phase. Participants must memorize the 30 objects. B) Recognition test phase. Participants must report which of the two objects was just memorized. 
Working memory task. Participants completed 250 trials of the working memory task; see Figure 2A. Each trial started with a white fixation screen that contained a black central fixation cross measuring $1 \times 1^{\circ}$, presented for $520 \mathrm{~ms}$. This was followed by a blank white screen for $200 \mathrm{~ms}$. Next, a memory array appeared for $400 \mathrm{~ms}$, which consisted of three objects from the pool of 2,152 object images, randomly chosen without replacement for each trial. The memory array never contained ACS or nonACS objects. Each object subtended $3 \times 3^{\circ}$, and randomly appeared in one of six possible locations; the six locations were equally spaced along an invisible centrally located circle with a $5^{\circ}$ radius. The objects disappeared for $800 \mathrm{~ms}$, and then one object appeared in one of the three original memory array locations. This single probe object was either the same object from that specific location in the memory array (requiring a "same" response by pressing the "s" key; $40 \%$ of trials), or a different object (requiring a "different" response by pressing the "d" key; $60 \%$ of trials). Different objects could be one of the 30 ACS objects (ACS probe, $20 \%$ of trials), one of the 30 nonACS objects (nonACS probe, $20 \%$ of trials), or from the pool of 2,152 object (novel probe, $20 \%$ of trials). Because we were primarily interested in examining RTs in this task, participants had only 1,500 ms to make a response, and were encouraged to respond as quickly and accurately as possible. If participants made an incorrect response, or failed to respond in time, a $500 \mathrm{~Hz}$ error tone sounded for $50 \mathrm{~ms}$. 


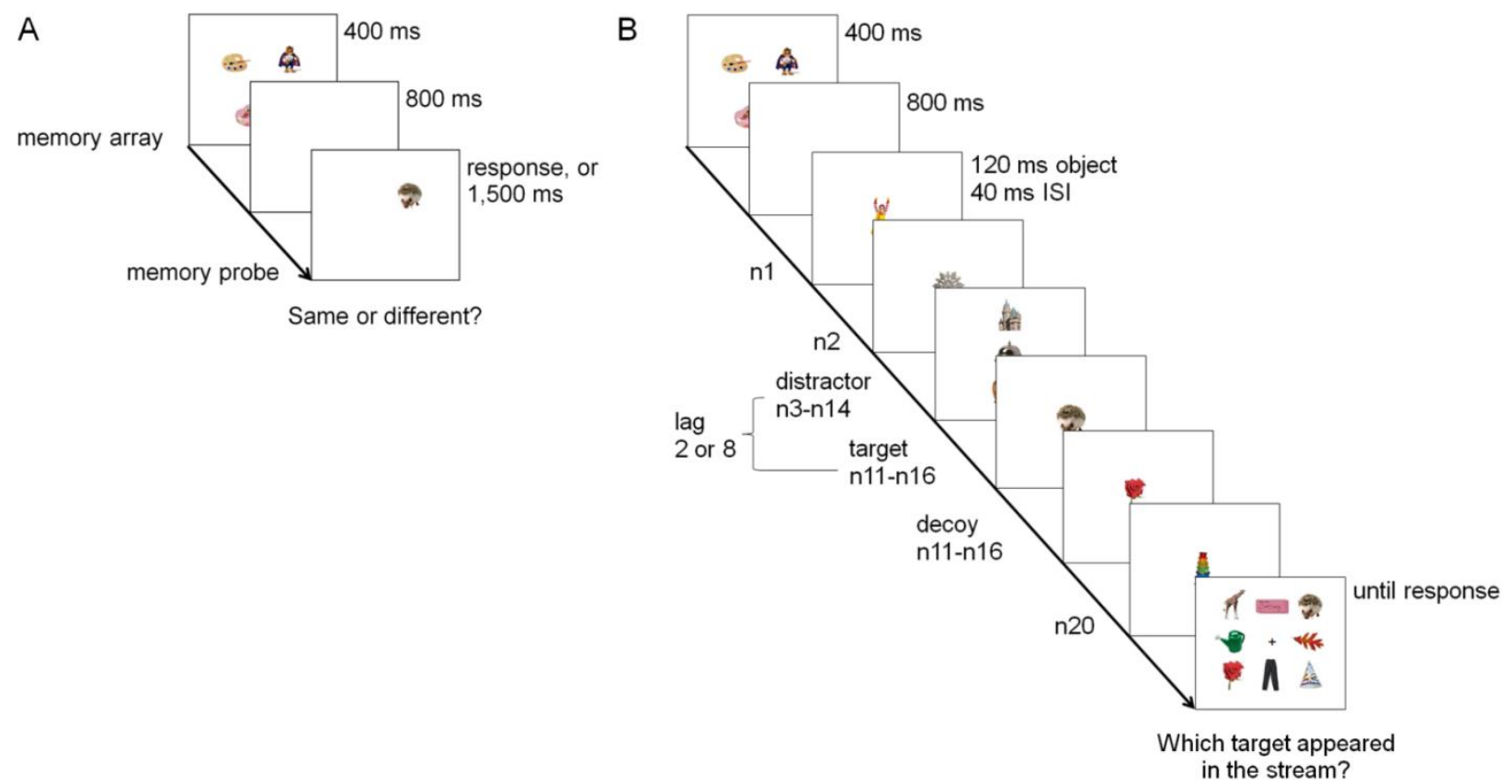

Figure 2. Sample trial sequences for Experiments 1 and 2. A) Trial sequence for the working memory task. Participants must report whether the object that appears is the same as, or different than, the object that was just in that location on the screen. B) Trial sequence for the spatial blink task. Participants must select on the final response screen, the target (ACS) object that appeared in the stream.

Spatial blink task. Randomly mixed with the working memory trials, participants completed 192 trials of a spatial blink task (Giammarco et al., 2016); see Figure 2B. Participants were instructed to keep their gaze on the middle of the screen and to look for one of the 30 ACS objects in the rapid stream of objects. Each trial started identically to trials from the working memory task, but a spatial blink stream was presented after the 800 ms memory delay rather than a memory probe. During the stream, 20 random objects that each subtended $3.4 \times 3.4^{\circ}$ were presented centrally one at a time for 120 $\mathrm{ms}$, separated by an inter-stimulus interval of $40 \mathrm{~ms}$. Both an ACS target object and a nonACS decoy object appeared within the stream, and could be at position $11-16$. Notably, two distractor objects were presented either two or eight objects before the target (i.e., distractor-target lags of 2 or 8 ), centered $4.24^{\circ}$ above and below the central stream. Distractor objects subtended $4.58 \times 4.58^{\circ}$, and appeared and disappeared with the central stream object. One distractor was the critical distractor, and was either an 
ACS object or a nonACS object. The critical distractor was never the ACS target object or the nonACS decoy object for that trial. The other distractor was a randomly selected novel object that was different from all other novel objects presented during the trial. Critical distractor type (ACS or nonACS) and distractor-target lag (2 or 8) were fully crossed and occurred with equal frequency for each participant.

At the end of the stream, there was a response screen with a $1 \times 1^{\circ}$ black central fixation cross and eight $3 \times 3^{\circ}$ objects arranged in a $10 \times 10^{\circ}$ grid around fixation. Participants were instructed to select the ACS object that had appeared in the spatial blink stream on the current trial by pressing the corresponding key on the keyboard numeric keypad. The objects presented on the response screen always included: the ACS target object, the nonACS decoy object, three ACS objects that did not appear during the stream, and three nonACS objects that did not appear during the stream. The critical distractor object, which should be ignored, was never included as an option on the response screen in order to prevent participants from attempting to strategically use distractor objects. The other objects were included on the response screen to control for exposure (ACS and nonACS objects were shown equally as often for the duration of the spatial blink task) and to ensure participants selected only the ACS target object that appeared within the stream. There was no time limit for response selection, and participants were encouraged to be as accurate as possible for this task. If the participant selected the wrong object, a $500 \mathrm{~Hz}$ tone was played for $50 \mathrm{~ms}$.

Statistical analyses. As is conventional, we predominately conducted inferential statistical analyses using null-hypothesis significance tests. We also sometimes made use of Bayes factors (BFs), in particular when evaluating how to interpret null effects (for a review of BFs, see Morey \& Rouder, 2011). Briefly, a $B F_{01}$ compares the null model against the alternative, with a $\mathrm{BF}_{01}$ value greater than 1 indicating evidence in favour of the null model (i.e., no difference between conditions), and less than 1 indicating evidence in favour of the alternative model. $\mathrm{A} \mathrm{BF}_{10}$ compares the alternative model against the null model (i.e., $\mathrm{BF}_{10}=1 / \mathrm{BF}_{01}$ ). For readability, we report whichever Bayes factor $\left(\mathrm{BF}_{10}\right.$ or $\mathrm{BF}_{01}$ is greater than 1$)$; $\mathrm{BF}$ values from 1-3 indicate anecdotal 
(insufficient) evidence, 3-10 moderate evidence, and greater than 10 strong evidence. We calculated Bayes factors using JASP version 0.8.3.1 (JASP-Team, 2017), using the default priors. JASP was also used for null-hypothesis significance tests.

\section{Results and Discussion}

Memory training task. As is common with such memory training tasks (Giammarco et al., 2016; Wolfe, 2012), most participants achieved the criterion level of accuracy in their first two blocks of memory training, which was the minimum possible amount of training. Only one participant required a third block, resulting in an average of 2.03 blocks for participants to complete the memory training task. Average accuracy (proportion of correct trials) was $97.8 \%$, suggesting that participants were able to successfully commit the 30 ACS objects to memory.

Spatial blink task. Accuracy data for the spatial blink task are plotted in Figure 3. A 2 (distractor-target lag: 2 vs. 8) $\times 2$ (distractor type: ACS vs. nonACS) within-subjects ANOVA was conducted to assess differences in accuracy. This analysis revealed statistically significant main effects of distractor-target lag, $F(1,28)=42.0, p<.001, \eta_{p}^{2}$ $=.600$, and distractor type, $F(1,28)=8.83, p=.006, \eta_{p}^{2}=.240$, and a non-significant interaction, $F(1,28)=2.90, p=.100, \eta_{p}^{2}=.094$. 


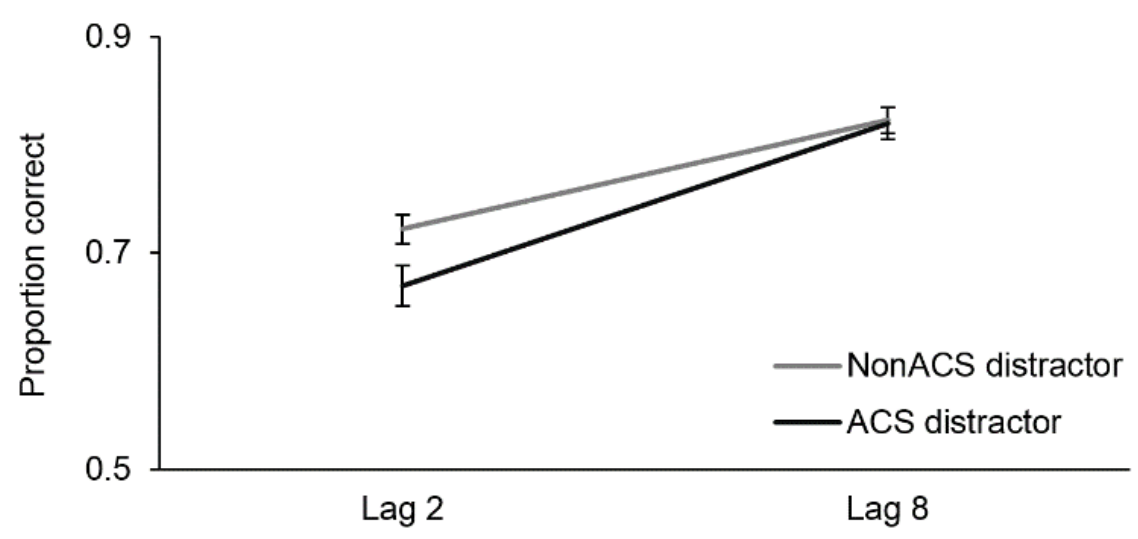

Figure 3. Accuracy data for the spatial blink (SB) task for Experiment 1, revealing a modulation of attentional capture (i.e., a greater impairment of target detection at lag 2 by ACS distractors than nonACS distractors). Error bars in this figure, and all subsequent figures, are corrected (Morey, 2008) within-subject standard errors (Cousineau, 2005).

Two planned paired-samples $t$-tests were conducted to determine whether participants exhibited contingent capture at lag 2 , but not at lag 8 . As predicted, accuracy for the lag 2 ACS distractor condition was significantly lower than for the nonACS distractor condition, $t(28)=2.58, p=.008$, one-tailed; and lag 8 accuracy for the ACS distractor condition was not significantly different from the nonACS distractor condition, $t(28)=$ $0.201, p=.842$. The Bayesian paired-samples $t$-tests provided moderate evidence for the alternative model for the difference at lag $2, \mathrm{BF}_{10}=6.26$, one-tailed, and moderate evidence for the null model at lag $8, \mathrm{BF}_{01}=4.97$. In addition to these planned tests, we also conducted a post hoc paired-samples $t$-test to determine whether accuracy differed for the nonACS distractor condition at lag 2 versus at lag 8 , as it appeared that the nonACS objects may have captured attention (i.e., produced a spatial blink); this test was statistically significant, $t(28)=4.97, p<.001$. It is possible that nonACS objects captured attention because participants had to attend and respond to them when they appeared as probes in the working memory task, and thus they were somewhat task relevant. Regardless, we critically observed a modulation of the magnitude of capture by the ACS objects at lag 2, with ACS distractors producing a greater impairment than nonACS distractors. 
Working memory task. Reaction time data for the change trials of the working memory task are plotted in Figure 4; note that RTs on same trials are not relevant for measuring the intrusion effect. Reaction time data were trimmed of outliers; for each participant, trials with a RT greater than 2.5 standard deviations from the condition mean were removed (1.97\% of trials). Incorrect response trials (8.55\%) and timeout trials (3.68\%) were also excluded.

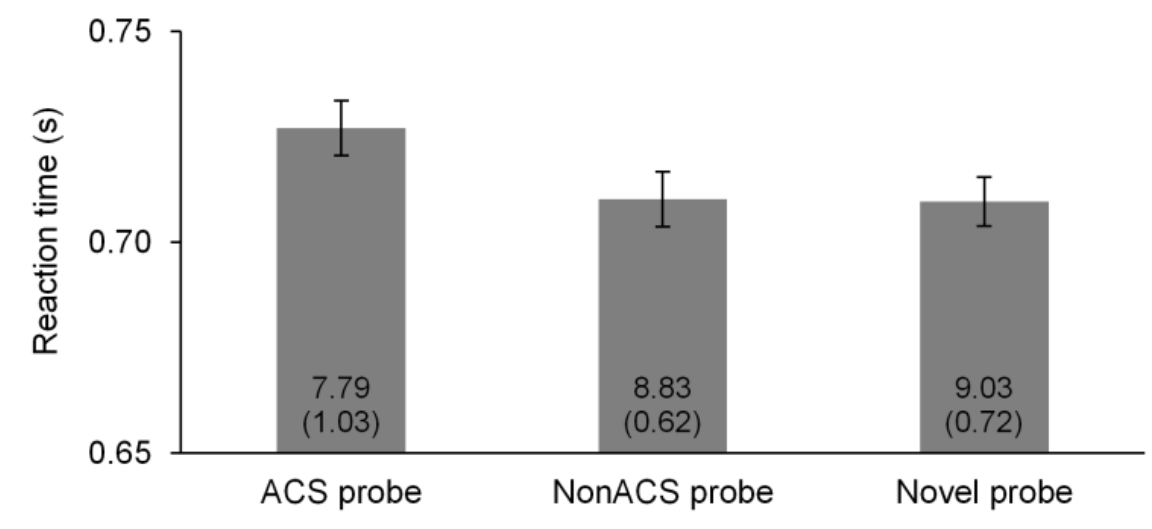

Figure 4. Reaction time data for the working memory task for Experiment 1. Numbers inside the bars indicate the error percentage (and corrected within-subject standard error; Morey, 2008) for each condition.

To assess the statistical significance of any differences in RTs across conditions, we conducted a 3-level one-way (ACS probe vs. nonACS probe vs. novel probe) withinsubjects ANOVA. This test revealed no statistically significant difference, $F(2,56)=$ 2.54, $p=.088, \eta_{p}{ }^{2}=.083$, and the Bayesian ANOVA provided anecdotal support for the null model, $\mathrm{BF}_{01}=1.37$. However, we also conducted two planned one-tailed pairedsamples $t$-tests to determine if the RTs for ACS probes were significantly slower than the other probes, which revealed statistically significant differences: nonACS probe, $t(28)=1.92, p=.032$; novel probe, $t(28)=1.875, p=.036$. The Bayesian pairedsamples $t$-tests provided anecdotal evidence to support the alternative model: $\mathrm{BF}_{10}=$ 
1.90 and 1.76, respectively. Overall, these results indicate that ACS probes produced an intrusion effect relative to nonACS probes and novel probes, suggesting that the ACS objects could be represented in ALTM.

Error rates are indicated using numerals in Figure 4. To assess speed-accuracy tradeoffs, the same 3-level ANOVA was conducted on error rates (proportion of trials where participants responded "same" instead of "different" on change trials), revealing no statistically significant differences, $F<1$. The Bayesian ANOVA provided moderate evidence to support the null model, $\mathrm{BF}_{01}=5.48$. Additionally, three paired-samples $t$ tests between the conditions revealed no statistically significant differences, all $p s>$ .340 , with moderate evidence to support the null model for each, all BFs $\mathrm{s}_{01}>3.21$. These results suggest that speed/accuracy trade-offs did not contribute to the observed differences in RTs.

\section{Summary}

The goal of Experiment 1 was to provide a preliminary assessment of the ALTM account by testing the prediction that, when participants adopt an LTM ACS, the objects that comprise the attentional set should be represented in ALTM. Consistent with this prediction, participants in the present experiment successfully adopted an attentional set for the 30 ACS objects, as evidenced by greater lag 2 ACS distractor costs in the spatial blink task relative to nonACS distractors, and these same ACS objects also produced an intrusion effect. While these results seem to provide preliminary support for the ALTM account, there is an alternative interpretation of these findings that must be assessed before drawing any conclusions. Namely, the observed modulations of the spatial blink and intrusion effect may be unrelated; the ACS objects may be represented in ALTM because that is part of the process through which ACSs are adopted, or they may be represented in ALTM for an unrelated reason, perhaps simply because they were recently memorized. One way to distinguish between these two interpretations would be to have participants memorize two separate lists of objects, and then only 
search for one of the lists in the spatial blink task; this is the approach we take in Experiment 2. Before moving to the next experiment, however, we can also compare these interpretations by testing for a correlation between each participant's ability to control attentional capture and the extent to which they represented ACS objects in ALTM. The results of this analysis are plotted in Figure 5, and reveal little to no relationship, $r=.092, p=.636, \mathrm{Cl}_{95}[-0.284,0.443]$. Indeed, the Bayesian correlation provided moderate evidence for the null model, $\mathrm{BF}_{01}=3.90$. Although the ACS manipulation in the present study modulated both attentional capture and ALTM, the lack of a correlation provides preliminary evidence that these modulations are not functionally related, and thus provide evidence against the ALTM account.

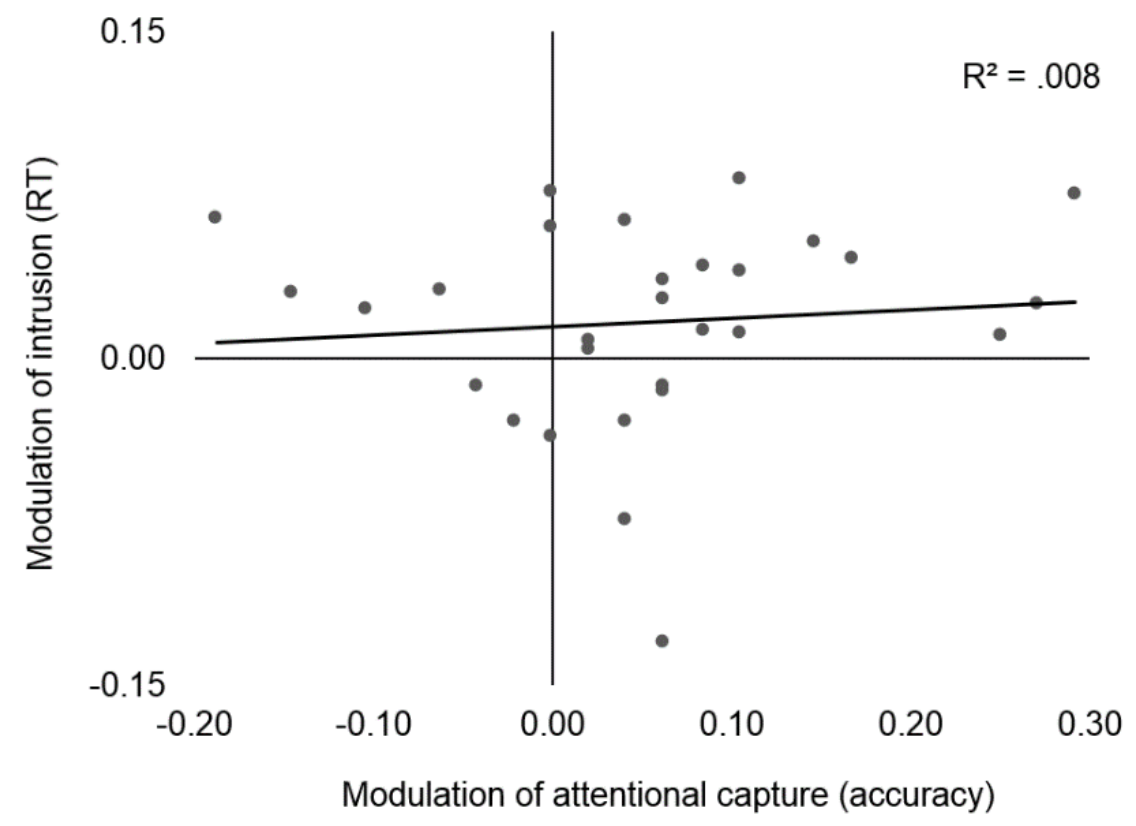

Figure 5. Correlation between individual differences in participants' ability to use an ACS to modulate attentional capture in the spatial blink task (nonACS distractor accuracy - ACS distractor accuracy), and to modulate the intrusion effect in the working memory task (ACS probe RT - nonACS probe RT) for Experiment 1. For both modulation measures, larger positive values indicate greater control. 


\section{Experiment 2}

The goal of Experiment 2 was to replicate and extend the findings from Experiment 1 by examining why ACS objects in that experiment produced greater intrusions than nonACS objects. In Experiment 1, these sets of objects differed in two ways: Unlike nonACS objects, 1) ACS objects were designated as targets in the spatial blink task, and 2) ACS objects were memorized during the training phase at the beginning of the experimental session. Might the difference in the intrusion effect be due to differences in training, and unrelated to ACSs? In Experiment 2, we controlled for differences in training by having participants memorize two separate lists of 15 objects, and then designated only one list of objects as the targets in the spatial blink task (i.e., the ACS objects). The other list served as the nonACS objects, thus equating training between ACS and nonACS objects. We have previously used this training manipulation to show that, of the two lists of studied objects, only the objects assigned as targets capture attention (Giammarco et al., 2016). What remains to be determined is whether there is also a difference in the working memory intrusions produced by ACS and nonACS objects, when training is controlled.

\section{Method}

Participants. A new sample of 32 undergraduate students from the University of Guelph (mean age 19.2 years, 22 females) participated in Experiment 2 for partial course credit. Three participants with error rates at $50 \%$ or higher on the spatial blink task were excluded from analyses. Thus, Experiment 2 had a final sample size of 29 participants.

Apparatus, stimuli, and procedure. All equipment and visual stimuli were identical to those in Experiment 1. There were three changes to the procedure for Experiment 2. First, in an attempt to remove fewer participants due to poor performance on the tasks, we had all participants complete a practice session before the experimental session. 
Second, at the end of the experimental session, we tested participants' memory to verify that they could still recognize both ACS and nonACS objects. Third, we altered training so that participants memorized two lists of 15 objects, rather than one list of 30 , and only one of the lists was designated as targets for the spatial blink task (ACS objects; the other as nonACS objects).

Practice session. The practice session was an abridged version of Experiment 2. Participants were shown ten objects from the pool of 2,152 object images, divided into two lists of five objects ("List A" and "List B"); participants passively viewed these objects one at a time, and then completed a recognition memory test where they viewed each of these ten practice objects and five novel objects one at a time, and responded whether the object was from List A, List B, or neither. One of the lists was then designated as the targets for the spatial blink task; participants then completed 24 spatial blink trials and 24 working memory trials, randomly mixed, before completing the main experiment. Because all stimuli were selected from the larger pool of 2,152 images, stimuli from the practice session were never assigned as either ACS or nonACS objects in the main experiment.

Memory training. For the memory training task, two lists of 15 objects were randomly selected without replacement from the pool of 125 object images ("List A" and "List B"). During the study phase of training, participants first saw the words "List A" on the screen, followed by the appearance of each List A object one at a time. This was followed by the words "List B" and then then presentation of the List B objects. After studying the objects, participants completed a memory test where they viewed each of these 30 objects and 15 novel objects (selected from the pool of 2,152 objects) one at a time, and reported whether the object was from List A, List B, or neither. Object presentation order was randomized for each study phase and for each recognition memory test phase. To ensure that participants had adequately learned each object and its source, participants completed the memory training task (both study and test phases) at minimum four times; participants had to achieve an accuracy of $90 \%$ or higher on the last two consecutive test phases (Giammarco et al., 2016; Wolfe, 2012). Upon 
completion of the memory training task, participants were instructed that objects from one of the two lists they had just studied would be the targets for the spatial blink task ("ACS objects"); the objects from the other list would therefore not be targets for the spatial blink task ("nonACS objects").

Working memory and spatial blink tasks. These two tasks were identical to Experiment 1, with the only difference being that List $A$ and List $B$ were assigned as the ACS and nonACS objects (assignment counterbalanced across participants). Participants completed 200 working memory trials and 168 spatial blink trials.

Final memory check. New for Experiment 2, at the end of the experiment, participants completed a memory check task in which they saw the 30 studied objects and 15 novel objects one at a time, and responded whether the object was from List A, List B, or neither. This was added to verify that participants remembered both lists of objects, as well as their sources, for the duration of the experiment.

\section{Results and Discussion}

Memory training and final memory check. All participants completed memory training in four blocks (the minimum). Average accuracy (proportion of trials in which the object's source was correctly identified) on the memory training was $94.0 \%$, suggesting that participants were able to commit the objects and their sources (i.e., List A, List B, or neither) to memory.

One participant did not complete the final memory check. Average accuracy on the memory check was $97.0 \%$. More specifically, average accuracy was $95.5 \%$ for ACS objects, $97.6 \%$ for nonACS objects, and $97.9 \%$ for novel objects; a paired-samples $t$ test revealed that recognition accuracy of ACS and nonACS objects was not statistically significantly different, $t(27)=1.43, p=.164$, and the Bayesian paired-samples $t$-test provided anecdotal evidence in support of the null model, $\mathrm{BF}_{01}=2.00$. Participants were able to recognize objects from both lists equally well. 
Spatial blink task. Accuracy data for the spatial blink task are plotted in Figure 6 . The 2 (distractor-target lag: 2 vs. 8) $\times 2$ (distractor type: ACS vs. nonACS) within-subjects ANOVA revealed statistically significant main effects of distractor-target lag, $F(1,28)=$ $34.3, \mathrm{p}<.001, \mathrm{\eta}_{\mathrm{p}}{ }^{2}=.550$, and distractor type, $F(1,28)=13.2, p<.001, \mathrm{\eta}_{\mathrm{p}}{ }^{2}=.321$, and a significant interaction $F(1,28)=4.79, p=.037, \eta_{p}^{2}=.146$.

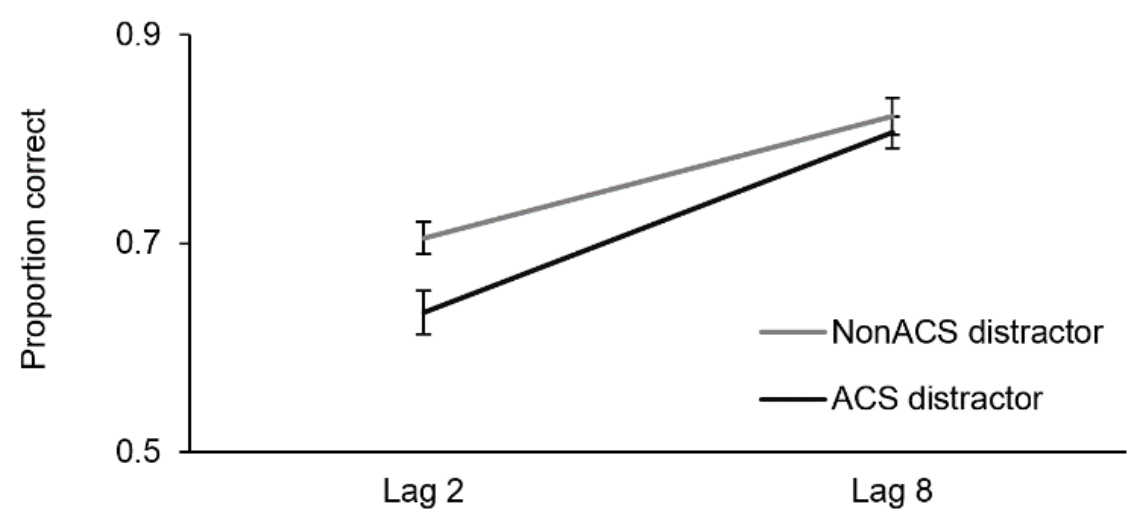

Figure 6. Accuracy data for the spatial blink (SB) task for Experiment 2, revealing a modulation of attentional capture (i.e., a greater impairment of target detection at lag 2 for ACS distractors than nonACS distractors).

Two planned paired-samples $t$-tests were conducted to determine whether participants exhibited contingent capture at lag 2, but not at lag 8 . As predicted, accuracy for the lag 2 ACS distractor condition was significantly lower than for the nonACS distractor condition, $t(28)=3.59, p<.001$, one-tailed; and accuracy for the lag 8 ACS distractor condition was not significantly different from the nonACS distractor condition, $t(28)=$ 1.06, $p=.297$. The Bayesian paired-samples $t$-tests provided strong evidence to support the alternative model for the difference at lag $2, \mathrm{BF}_{10}=54.6$, one-tailed, and moderate evidence for the null model for the difference at lag $8, \mathrm{BF}_{01}=3.03$. In addition to these planned tests, we also conducted a post hoc paired-samples $t$-test to determine whether accuracy differed for the nonACS distractor condition at lag 2 versus at lag 8 , as it appeared that the nonACS objects may have captured attention (i.e., produced a 
spatial blink); this test was statistically significant, $t(28)=4.45, p<.001$. Despite the fact that the nonACS distractors captured attention, we still observed a modulation of the magnitude of capture by the ACS objects at lag 2, with ACS distractors producing a greater impairment than nonACS distractors.

Working memory task. Reaction time data for the change trials of the working memory task are plotted in Figure 7. Data were trimmed of outliers; for each participant, trials with a RT greater than 2.5 standard deviations from the condition mean were removed $(1.83 \%$ of trials). Incorrect response trials $(8.85 \%)$ and timeout trials $(3.33 \%)$ were also excluded from RT analyses.

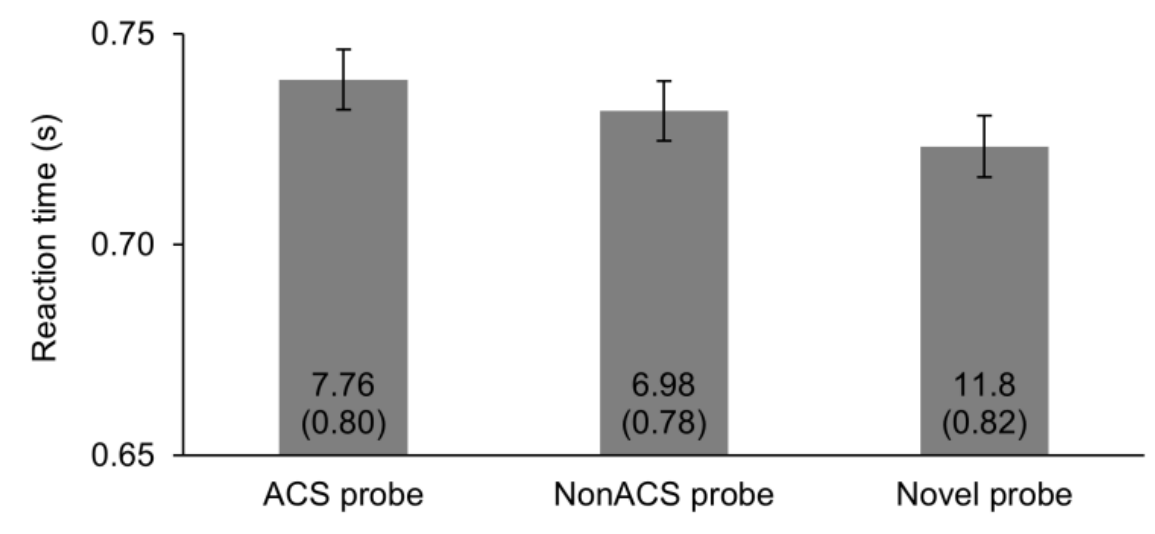

Figure 7. Reaction time data for the working memory task for Experiment 2. Numbers inside the bars indicate the error percentage for each condition.

The 3-level one-way (ACS probe vs. nonACS probe vs. novel probe) within-subjects ANOVA was not statistically significant, $F(2,56)=1.25, p=.295, \eta_{p}{ }^{2}=.043$, and the Bayesian ANOVA provided moderate evidence to support the null model, $\mathrm{BF}_{01}=3.54$. Two planned one-tailed paired-samples $t$-tests were also conducted to determine if the RTs for the ACS probe condition significantly differed from the nonACS probe condition, $t(28)=0.755, p=.228$, and the novel probe condition, $t(28)=1.55, p=.066$. The Bayesian one-tailed paired-samples $t$-tests provided anecdotal evidence for the null 
model, $\mathrm{BF}_{01}=2.56$ and anecdotal evidence for the alternative model, $\mathrm{BF}_{10}=1.07$ respectively. In addition to these planned tests, we also conducted a post hoc pairedsamples $t$-test to determine whether RTs differed between the nonACS probe and novel probe conditions, as it appeared that the nonACS objects may have exhibited an intrusion effect; this test was not statistically significant, $t(28)=0.834, p=.411$. The follow-up Bayesian paired-samples $t$-test provided moderate evidence for the null model, $\mathrm{BF}_{01}=3.68$.

Error rates are indicated using numerals in Figure 7. To assess speed-accuracy tradeoffs, the same 3-level (ACS probe, nonACS probe, novel probe) ANOVA was conducted on error rates (proportion of trials where participants responded "same" instead of "different" on change trials), revealing a statistically significant difference, $F(2,56)=$ $10.5, p<.001, \eta_{p}^{2}=.273$. The Bayesian ANOVA provided strong evidence to support the alternative model, $\mathrm{BF}_{10}=176$. As a follow-up, the three post-hoc paired-samples $t$ tests revealed a significant difference in error rates between the ACS probe and novel probe conditions, $t(28)=3.51, p=.002$, and between the nonACS probe and novel probe conditions, $t(28)=4.25, p<.001$. There was no significant difference in error rates between the ACS probe and nonACS probe conditions, $t(28)=0.703, p=.488$, and the Bayesian paired-samples $t$-test revealed moderate evidence to support the null model, $\mathrm{BF}_{01}=4.04$. These results suggest that speed/accuracy trade-offs may have contributed to the observed differences in RTs between conditions; participants made the most errors during the novel probe condition, which was the condition with the fastest RTs. Notably, these differences in accuracy are the opposite of what would be predicted by the ALTM account.

\section{Summary}

The primary goal of Experiment 2 was to determine whether the difference in the intrusion effects between ACS and nonACS objects in Experiment 1 would persist when controlling for training. Despite finding a robust difference in attentional capture by ACS 
and nonACS objects in the spatial blink task of Experiment 2, we did not observe a statistically significant difference in the intrusion effect for these objects; indeed, the Bayes factor somewhat favoured the null hypothesis $\left(\mathrm{BF}_{01}=2.56\right)$. Additionally, the RT difference between ACS and novel objects in the working memory task was relatively small, and only trending towards statistical significance. Finally, the only statistically reliable differences in performance across the conditions of the working memory task were on accuracy, and the observed pattern was inconsistent with ACSs objects being represented in ALTM (i.e., worse performance for novel probes than ACS probes). As in Experiment 1, we tested for a potential relationship between ACSs and ALTM by correlating each participant's ability to control attentional capture with the extent to which they represented ACS objects in ALTM. The results of this analysis are plotted in Figure 8 , and revealed little to no relationship, $r=-.239, p=.212, \mathrm{Cl}_{95}[-0.557,0.140]$. Indeed, this correlation is in the opposite direction compared to what the ALTM account would predict, and the Bayesian correlation provided anecdotal evidence for the null model, $\mathrm{BF}_{01}=2.06$. Thus, as with Experiment 1, these data do not support the ALTM account. 


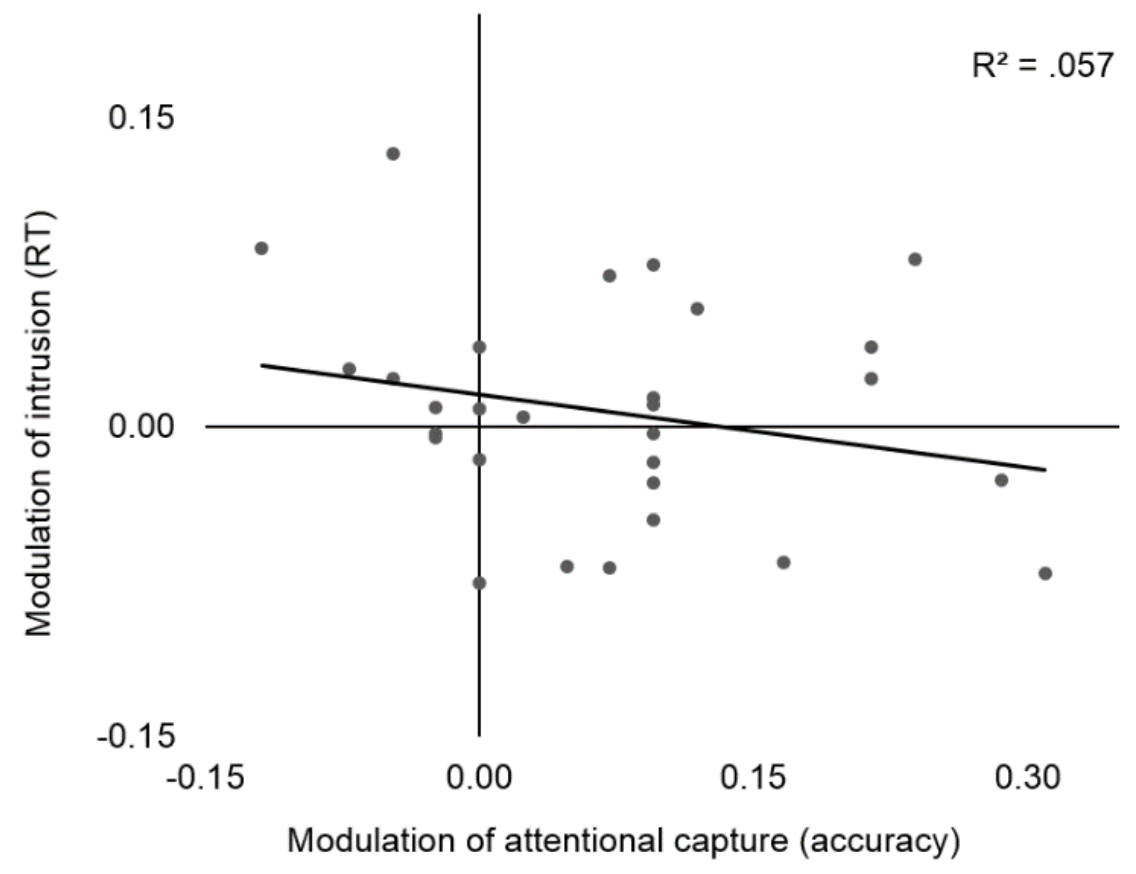

Figure 8. Correlation between individual differences in participants' ability to use an ACS to modulate attentional capture in the spatial blink task (nonACS distractor accuracy - ACS distractor accuracy), and to modulate the intrusion effect in the working memory task (ACS probe RT - nonACS probe RT) for Experiment 2. For both modulation measures, larger positive values indicate greater control.

Collapsing across Experiments 1 and 2, we can gain some insight into the observed results. On the one the hand, relative to novel objects, ACS objects do appear to produce a small but reliable intrusion effect, with a difference in RT of $16.6 \mathrm{~ms}, \mathrm{Cl}_{95}$ [2.94, 30.3], suggesting that the objects are, at least sometimes, represented in ALTM. This effect, however, appears to be epiphenomenal. There is no reliable difference between ACS and nonACS object intrusion effects when training is controlled, and individual differences in the ability to control attentional capture are unrelated to individual differences in the use of ALTM, $r=-.089, \mathrm{Cl}_{95}[-0.340,0.173], \mathrm{BF}_{01}=4.92$. Together, these results are consistent with the conclusion that ALTM does not serve a functional role in establishing LTM ACSs. 


\section{Chapter 3: Is Activated Long-term Memory Sufficient for Adopting a Long-term}

\section{Memory Attentional Control Setting?}

\section{Experiment 3}

To assess the ALTM account of LTM ACSs, the experiments so-far induced participants to adopt an ACS for complex visual objects, and then tested if those same objects were represented in ALTM. Experiments 3 and 4 tested the alternate direction of this relationship. Does inducing a participant to represent objects in ALTM cause the participant to adopt an ACS for those objects? If being in the activated state is the factor that determines whether or not LTM representations will bias attentional capture, then inducing participants to represent objects in ALTM should be sufficient for participants to adopt an ACS for those objects. Accordingly, in Experiment 3 we induced participants to represent objects in ALTM and then tested whether those objects captured attention to a greater extent than novel objects. To manipulate the contents of ALTM, we used a modified version of Oberauer's (2001) working memory task, in which participants first memorized two lists of visual objects, and were then provided a cue indicating which list would be probed, and which list could be ignored. Oberauer (2001) demonstrated that, because the objects from the ignored list are task irrelevant, participants do not maintain representations of those objects in working memory, as evidenced by the observation that manipulating the number (i.e., set size) of irrelevant objects to memorize has no effect on working memory RTs (Oberauer, 2001). Nevertheless, these objects still produce intrusion effects, indicating they are maintained in ALTM. Thus, in the present experiment, we first evaluated our ALTM manipulation by assessing set size and intrusion effects, and then tested the ALTM account by measuring capture on some trials using the spatial blink. For the spatial blink task, participants were provided a list of three target objects to search for, and we can expect participants to adopt an ACS for these objects. Critically, however, if representation in ALTM is the factor that determines whether an LTM representation is part of an ACS or not, then the irrelevant objects from 
the working memory task should also be incorporated into the ACS, and produce greater capture in the spatial blink task than novel objects.

\section{Method}

Participants. A new sample of 37 undergraduate students from the University of Guelph (mean age 19.5 years, 29 females) participated in Experiment 3 for partial course credit. Multiple participants had to be excluded from analyses due to the difficulty of the two tasks; this included three participants with error rates at $40 \%$ or higher on the working memory task, two participants with error rates at $40 \%$ or higher on the spatial blink task, and one participant who did not complete the experiment. Thus, Experiment $3 \mathrm{had}$ a final sample size of 31 participants. Notably, including all participants in the analyses reported below does not alter the pattern of statistically significant effects.

Apparatus, stimuli, and procedure. All equipment and visual stimuli were identical to those in Experiment 1. As with Experiments 1 and 2, all trials in Experiment 3 started with a working memory task, and ended with either a memory probe or a spatial blink trial. However, there were two main procedural changes for Experiment 3. First, the working memory task was redesigned to manipulate the contents of ALTM. Second, the targets for the spatial blink task were three objects selected randomly without replacement from the pool of 125 visual objects. Given the reduction in the number of spatial blink targets, we did not have participants complete memory training or memory test tasks; rather, the three objects were displayed concurrently at the beginning of the experimental session, and participants were instructed to memorize them for use in the spatial blink task.

Working memory task. Participants completed 144 trials of a modified version of Oberauer's (2001) modified Sternberg task; see Figure 9. Each trial started with a white fixation screen that contained a black central fixation cross measuring $1 \times 1^{\circ}$, presented for 1,000 ms. Next, a memory array appeared, which consisted of two lists that each 
contained either one or three objects per trial. One list was presented $10^{\circ}$ above the center of the screen, and was presented in a blue rectangular frame. The other list was presented $10^{\circ}$ below the center of the screen, and was presented in a red rectangular frame. Both frames were $5^{\circ}$ high, $30^{\circ}$ wide, with lines that were $0.04^{\circ}$ thick. Each object subtended $3 \times 3^{\circ}$ and appeared in one of three possible locations within the rectangular frame: $10^{\circ}$ to the left of center, central, or $10^{\circ}$ to the right of center. Objects and frames during the memory array were presented for 1,000 ms per object. For example, on a trial with one object in each frame, the memory array was presented for 2,000 ms. The memory array was followed by an 800 ms memory delay, which was followed by a cue screen. On the cue screen, a rectangular frame $\left(5^{\circ}\right.$ high, $30^{\circ}$ wide, lines $0.04^{\circ}$ thick) was centrally presented. The cue was randomly presented in red or blue, and indicated to participants which memory list would be probed at the end of the trial (i.e., the relevant list) and, consequently, which list was now irrelevant. This cue was $100 \%$ valid. Oberauer (2001) reported that, for young adults, within 1,000 ms the irrelevant items were no longer represented in working memory. As we changed the stimuli used from words to complex visual objects, to be conservative we presented the cue for $2,400 \mathrm{~ms}$ before adding the $3 \times 3^{\circ}$ memory probe to the display. The probe object could be: a relevant list object (relevant probe, $50 \%$ of trials, requires a "yes" response), an irrelevant list object (irrelevant probe, $25 \%$ of trials, requires a "no" response), or a novel object (novel probe, $25 \%$ of trials, requires a "no" response). All objects for each trial were randomly assigned without replacement from the pool of 2,152 objects images. Participants had 1,600 ms to respond whether the probe had been present in the relevant list; if participants made an incorrect response, or failed to respond in time, a $500 \mathrm{~Hz}$ error tone sounded for $50 \mathrm{~ms}$. The levels of relevant list set size (1 vs. 3), irrelevant list set size ( 1 vs. 3 ), and probe type (relevant vs. irrelevant vs. novel) were fully crossed for each participant. 

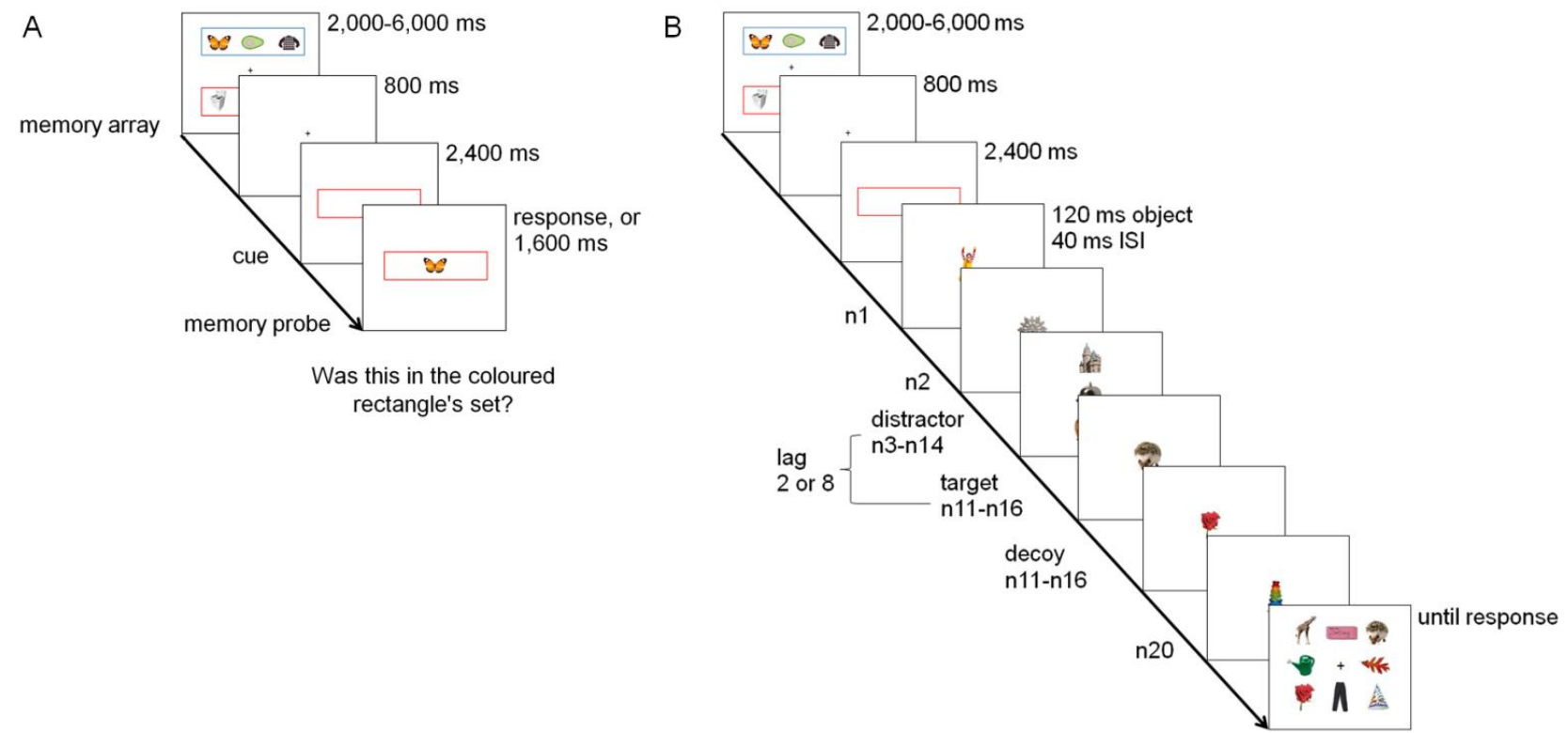

Which target appeared in the stream?

Figure 9. Sample trial sequences for Experiments 3 and 4. A) Trial sequence for the working memory task. Participants must report whether the object that appears was located in the cued coloured rectangle's set. B) Trial sequence for the spatial blink task. On the final response screen, participants must select the target that had appeared in the stream.

Spatial blink task. Randomly mixed with the working memory task, participants completed 144 spatial blink trials with a similar methodology as in Experiments 1 and 2. See Figure 9B. Each trial started identically to trials from the working memory task, but the memory probe was replaced with a spatial blink stream. To ensure no overlap among the objects used within a given trial, all non-target objects were selected from the pool of 2,152 images without replacement. The critical distractor on the trial was either an object from the irrelevant list in the memory task, or a novel object. The objects presented on the response screen always included: the target object that appeared in the stream; the other two target objects that did not appear in the stream; one of the novel objects from the stream, which served as the decoy object; and four novel objects not present in the stream. Otherwise, the spatial blink component of a trial was identical to that from Experiments 1 and 2. Critical distractor type (irrelevant or 
novel) and distractor-target lag (2 or 8) were fully crossed and appeared with equal frequency for each participant.

\section{Results and Discussion}

Working memory task. Data were trimmed of outliers; for each participant, trials with a RT greater than 2.5 standard deviations from the condition mean were removed (1.34\% of trials). Incorrect response trials (15.41\%) and timeout trials (6.00\%) were also excluded from RT analyses.

As with Oberauer (2001), we examined the effects of set size and probe type using separate analyses; see Figures 10 and 11 . Starting with set size, we collapsed across probe type and conducted a 2 (relevant set size: 1 vs. 3) $\times 2$ (irrelevant set size: 1 vs. 3 ) within subjects ANOVA on RTs. The resulting two-way interaction was not statistically significant, $F<1, \mathrm{BF}_{01}=3.15$. The main effect of relevant set size was statistically significant, $F(1,30)=228, p<.001, \eta_{p}^{2}=.884$, consistent with the conclusion that participants stored relevant objects in working memory; that is, the more relevant objects they were asked to remember, the longer it took them to make working memory judgements (Sternberg, 1966). Critically, the effect of irrelevant set size was nonsignificant, $F(1,30)=0.432, p=.516, \mathrm{n}_{\mathrm{p}}^{2}=.014$, and the Bayesian ANOVA moderately favoured the null model, $\mathrm{BF}_{01}=4.92 ; \mathrm{RTs}$ did not differ when participants were asked to remember one or three irrelevant objects, suggesting that irrelevant objects were not represented in working memory. 


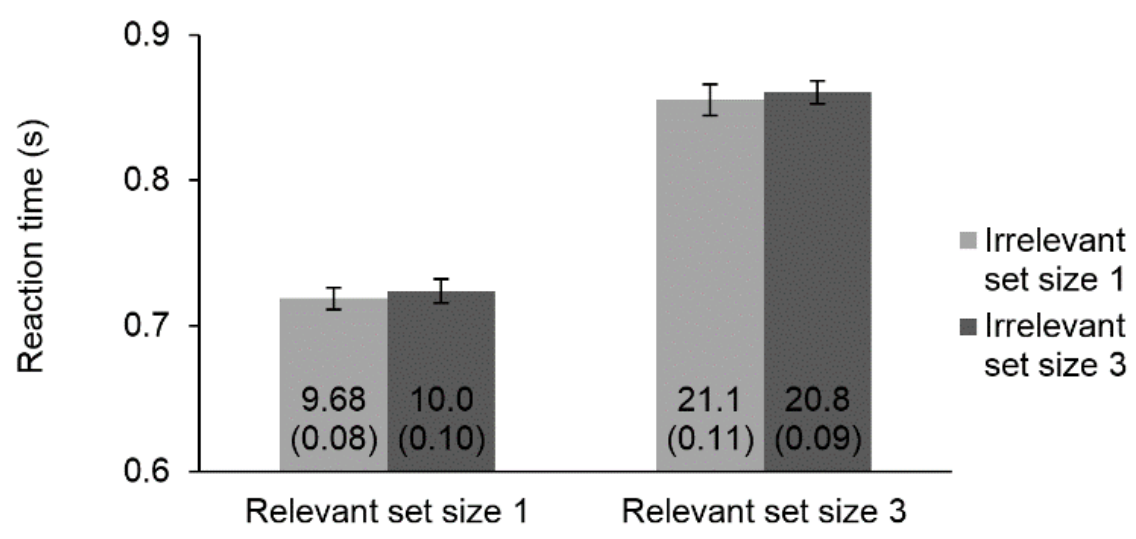

Figure 10. Set size reaction time data for Experiment 3's working memory task. Numbers inside the bars indicate the error percentage for each condition. Participants exhibited a relevant set size effect, but not an irrelevant set size effect.

In order to examine whether the irrelevant objects were represented in ALTM, we collapsed across relevant and irrelevant set sizes and examined the intrusion effect by comparing RTs for irrelevant probes to novel probes using a one-tailed planned pairedsamples $t$-test; see Figure 11. Participants took significantly longer to respond to irrelevant probes than to novel probes, $t(30)=7.35, p<.001$. This intrusion effect indicates that the working memory task successfully induced participants to represent irrelevant list objects in ALTM. 


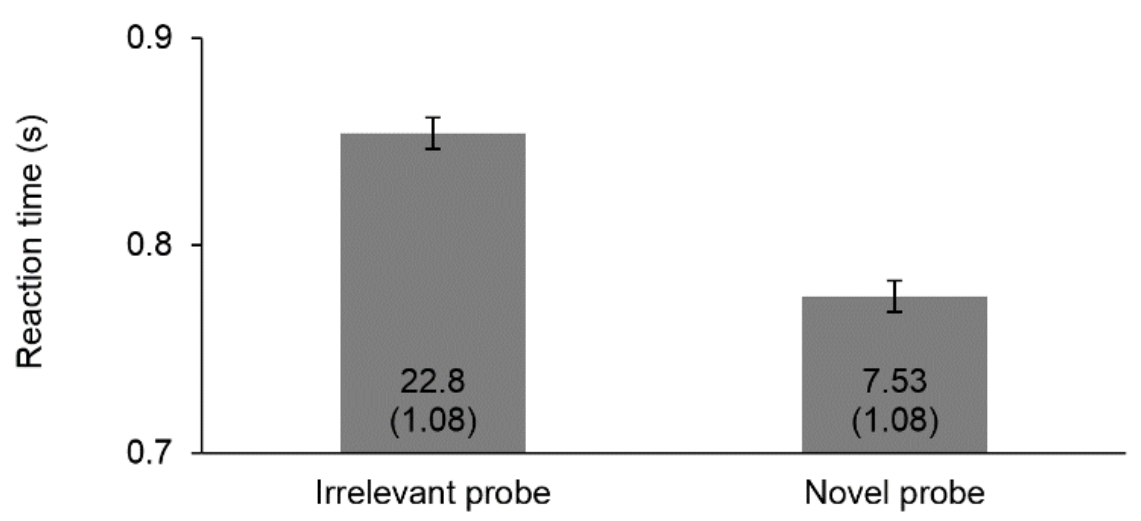

Figure 11. Reaction time data for two probe types for Experiment 3's working memory task. Participants exhibited an intrusion effect (longer reaction times to irrelevant probes than to novel probes). Numbers inside the bars indicate the error percentage for each condition.

Error rates are indicated using numerals in Figures 10 and 11. To assess speedaccuracy trade-offs for the conditions of interest, the same $2 \times 2$ ANOVA and $t$-test were run on error rates. The ANOVA revealed no statistically significant interaction, $F<1$, $\mathrm{BF}_{01}=4.14$, or irrelevant set size effect, $F<1, \mathrm{BF}_{01}=5.19$, but a statistically significant relevant set size effect, $F(1,30)=98.2, p<.001, \mathrm{n}_{\mathrm{p}}{ }^{2}=.766$. Additionally, the pairedsamples $t$-test revealed significantly more errors for the irrelevant probe condition than for the novel probe condition, $t(30)=10.0, p<.001$. Importantly, for both instances of significant differences in error rates, participants made more errors for the slower of the two conditions, thus it is unlikely that the observed differences in RTs were due to speed/accuracy trade-offs.

Spatial blink task. Accuracy data for the spatial blink task are plotted in Figure 12. A 2 (distractor-target lag: 2 vs. 8) $\times 2$ (distractor type: irrelevant vs. novel) within-subjects ANOVA was conducted to assess differences in accuracy. This analysis yielded a nonsignificant main effect of distractor-target lag, $F(1,30)=0.057, p=.812, \eta_{p}{ }^{2}=.002$, a non-significant main effect of distractor type, $F(1,30)=0.430, p=.517, \eta_{p}{ }^{2}=.014$, and a significant interaction, $F(1,30)=9.16, p=.005, \eta_{p}{ }^{2}=.234$. 


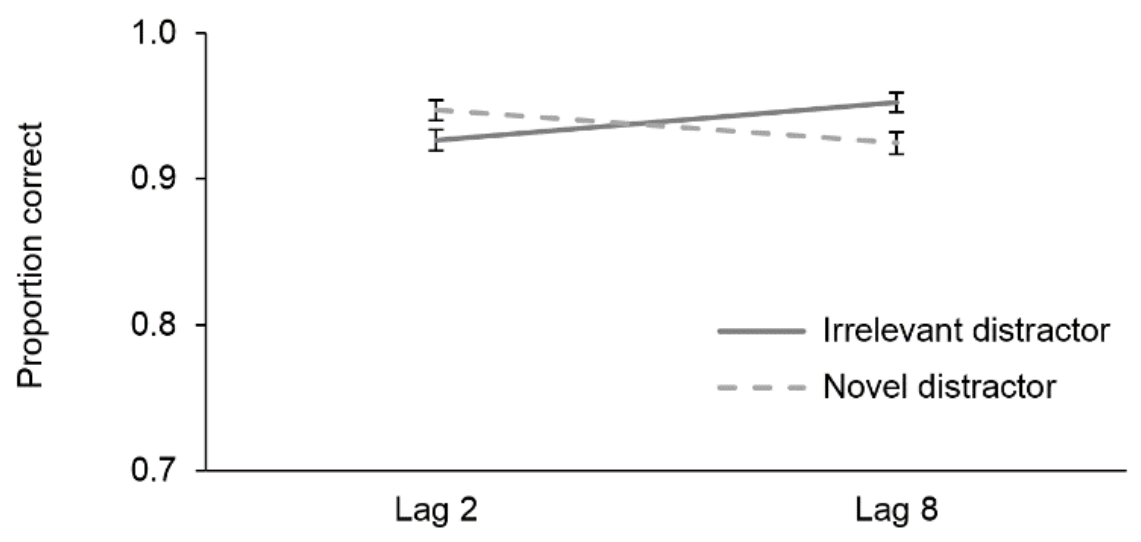

Figure 12. Accuracy data for the spatial blink (SB) task for Experiment 3 , revealing no contingent capture of the irrelevant distractor objects.

Two planned paired samples $t$-tests were conducted to determine whether participants exhibited contingent capture at lag 2, but not at lag 8. Accuracy for the lag 2 irrelevant distractor condition was significantly lower than for the novel distractor condition, $t(30)=$ 2.12, $p=.021$, one-tailed; surprisingly, lag 8 accuracy for the irrelevant distractor condition was also significantly different from the novel distractor condition $t(30)=2.88$, $p=.007$. To investigate these differences further, two Bayesian $t$-tests were conducted, revealing anecdotal evidence to support the alternative model for a difference at lag 2 , $\mathrm{BF}_{10}=2.63$, one-tailed, and moderate evidence to support the alternative model for a difference at lag $8, \mathrm{BF}_{10}=5.86$.

While the statistically significant drop in accuracy at lag 2 is consistent with the conclusion that representing irrelevant items in ALTM caused those items to capture attention, it is important to note that the remainder of the observed results are inconsistent with this interpretation. This account cannot explain the significant difference in accuracy at lag 8. As well, the differences in accuracy at both lag 2 and lag 8 are small at only $2.06 \%$ and $2.78 \%$, respectively, especially in comparison to previous spatial blink tasks, where ACS-matching distractors at lag 2 caused decreases in accuracy of $10-30 \%$ compared to novel distractors (Folk et al., 2002; Giammarco et al., 2016). Indeed, while we did not include a truly novel distractor condition for Experiments 
1 and 2 reported here, and even though both ACS and nonACS distractors captured attention in these experiments, the modulations of capture by the ACS manipulation were more than double (Experiment 1, 5.24\%) and triple (Experiment 2, 7.14\%) the magnitude of the effect observed in Experiment 3. Taking these results together, we tentatively conclude that the small, but statistically significant, differences observed in the present experiment likely reflect type I errors, and that irrelevant objects produced little to no attentional capture. To bolster this conclusion, we re-examined these conditions in Experiment 4.

\section{Summary}

The goal of Experiment 3 was to closely follow Oberauer's (2001) working memory methodology to induce participants to represent complex visual objects in ALTM, and then to test whether these representations spontaneously form an ACS. While participants in the present experiment successfully represented the irrelevant list objects in ALTM, as demonstrated by the non-significant irrelevant set size effect and the significant intrusion effect, the irrelevant list objects did not produce the type of attentional capture effects typically observed in spatial blink tasks. Thus, it would appear that representing stimuli in ALTM is not sufficient for those same stimuli to form an ACS. Despite observing minimal evidence for capture by irrelevant items, to mirror the Experiment 1 and 2 analyses we once again tested for relationships between individual differences in the intrusion effect and contingent capture; see Figure 13. This analysis once again revealed little to no relationship, $r=-.341, p=.061, \mathrm{Cl}_{95}[-0.620,0.016], \mathrm{BF}_{10}$ $=1.20$, and, indeed, was in the opposite direction of what would be expected. Together, the results of Experiment 3 are inconsistent with the ALTM account. 


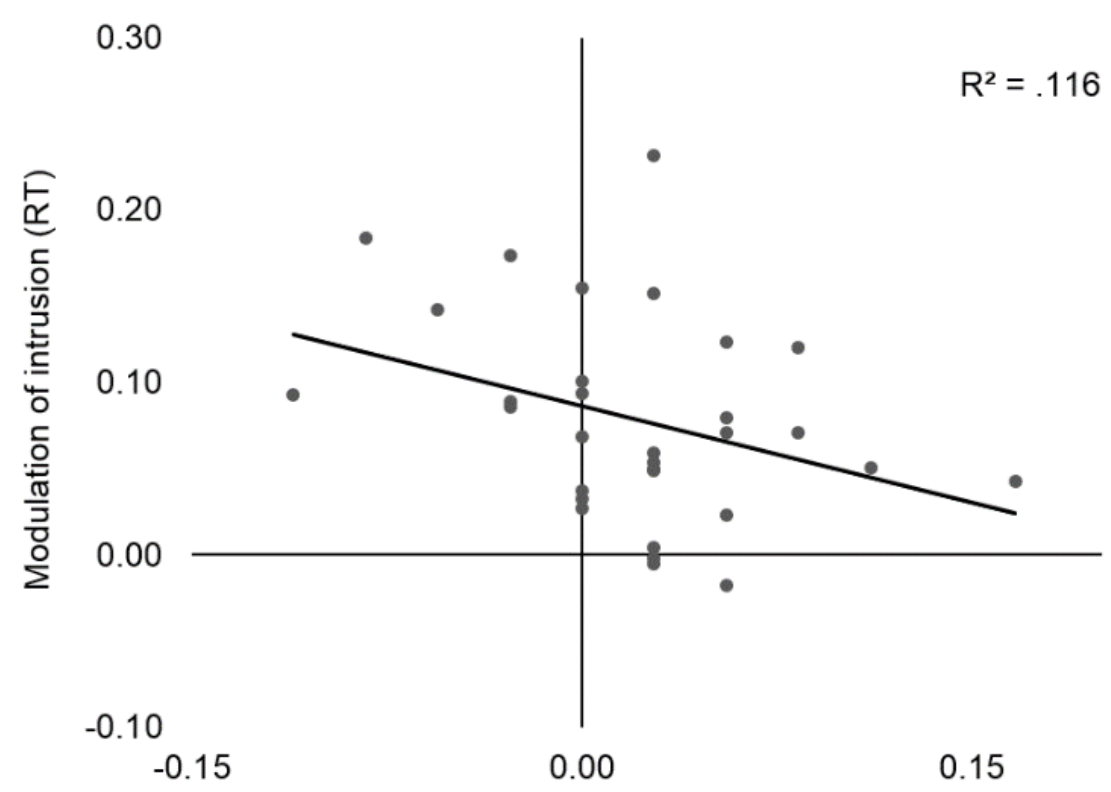

Figure 13. Correlation between individual differences in participants' ability to use an ACS to modulate attentional capture in the spatial blink task (novel distractor accuracy - irrelevant distractor accuracy), and to modulate the intrusion effect in the working memory task (irrelevant probe RT - novel probe RT) for Experiment 3. For both modulation measures, larger positive values indicate greater control.

\section{Experiment 4}

The goal of Experiment 4 was to replicate the results of Experiment 3, and to extend them by including an ACS-matching distractor condition to provide a baseline measure of typical attentional capture during a spatial blink task. Accordingly, in addition to irrelevant and novel distractor conditions, the spatial blink task in Experiment 4 included a target distractor condition. Participants memorized six target objects at the beginning of the experiment that they had to search for during the spatial blink task, and occasionally these target objects would appear as distractors. By virtue of searching for these target objects, the objects should form an ACS and exhibit typical contingent capture during the spatial blink task, thus, providing a suitable baseline for assessing capture by irrelevant list objects. 


\section{Method}

Participants. A new sample of 28 undergraduate students from the University of Guelph (mean age 18.3 years, 9 females) participated in Experiment 4 for partial course credit. Two participants with error rates at $40 \%$ or higher on the working memory task and one participant an error rate at $40 \%$ or higher on the spatial blink task were excluded from analyses Thus, Experiment 4 had a final sample size of 25 participants.

Apparatus, stimuli, and procedure. All equipment and visual stimuli were identical to those in Experiment 3. For Experiment 4, participants studied six objects at the beginning of the experiment, which were used as targets for the spatial blink task; these objects were randomly selected without replacement from the pool of 125 visual objects. The increase in the number of target objects from three to six for Experiment 4 was necessary so that, on spatial blink trials with a target distractor, the selection screen at the end of the trial could still include three targets objects without including the distractor.

Working memory and spatial blink tasks. There were 144 working memory trials and 144 spatial blink trials. These two tasks were identical to Experiment 3, with the only difference being the addition of a "target distractor" condition in the spatial blink task.

\section{Results and Discussion}

Working memory task. Data were trimmed of outliers; for each participant, trials with a RT greater than 2.5 standard deviations from the condition mean were removed (1.42\% of trials). Incorrect response trials (18.5\%) and timeout trials (4.67\%) were also excluded from RT analyses.

Again, we collapsed across probe type and conducted a 2 (relevant set size: 1 vs. 3 ) $\times 2$ (irrelevant set size: 1 vs. 3 ) within subjects ANOVA on RTs; see Figure 14. Similar to Experiment 3 , the ANOVA revealed a non-significant interaction, $F<1, \mathrm{BF}_{01}=3.01$, a 
significant main effect of relevant set size, $F(1,24)=86.4, p<.001, \eta_{\mathrm{p}}{ }^{2}=.783$, and a non-significant main effect of irrelevant set size, $F(1,24)=1.43, p=.244, \eta_{p}{ }^{2}=.056$. Additionally, the Bayesian ANOVA moderately favoured the null model for the main effect of irrelevant set size, $\mathrm{BF}_{01}=4.12$, suggesting that irrelevant objects were not represented in working memory.

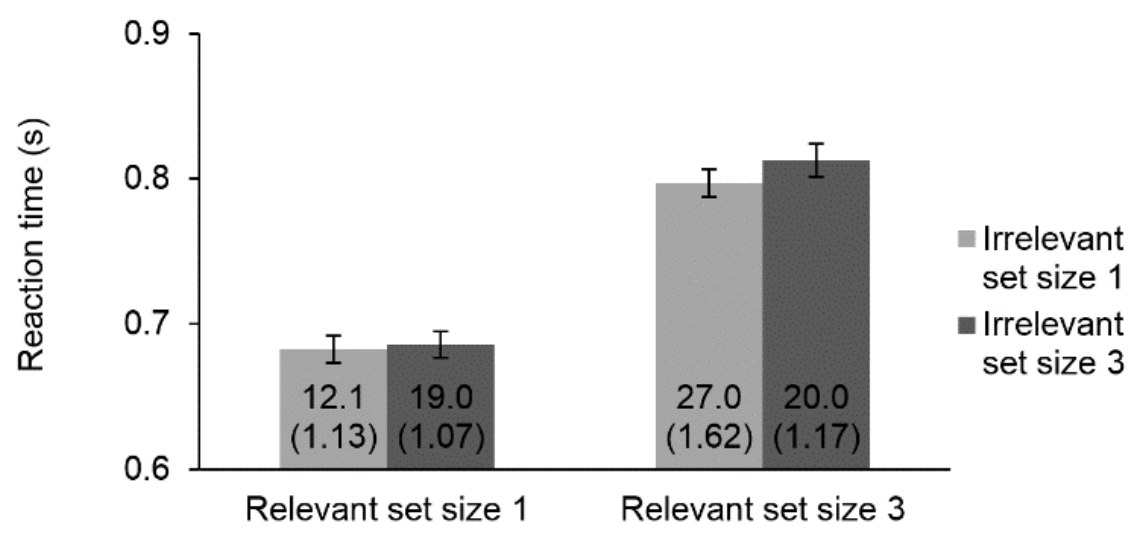

Figure 14. Set size reaction time data for Experiment 4's working memory task. Numbers inside the bars indicate the error percentage for each condition. Participants exhibited a relevant set size effect, but not an irrelevant set size effect.

In order to examine whether the irrelevant objects were represented in ALTM, we again collapsed across relevant and irrelevant set sizes to examine the intrusion effect; see Figure 15. The one-tailed planned paired-samples $t$-test revealed longer RTs when participants responded to irrelevant probes than novel probes, $t(24)=4.13, p<.001$. This intrusion effect indicates that the working memory task successfully induced participants to represent irrelevant list objects in ALTM. 


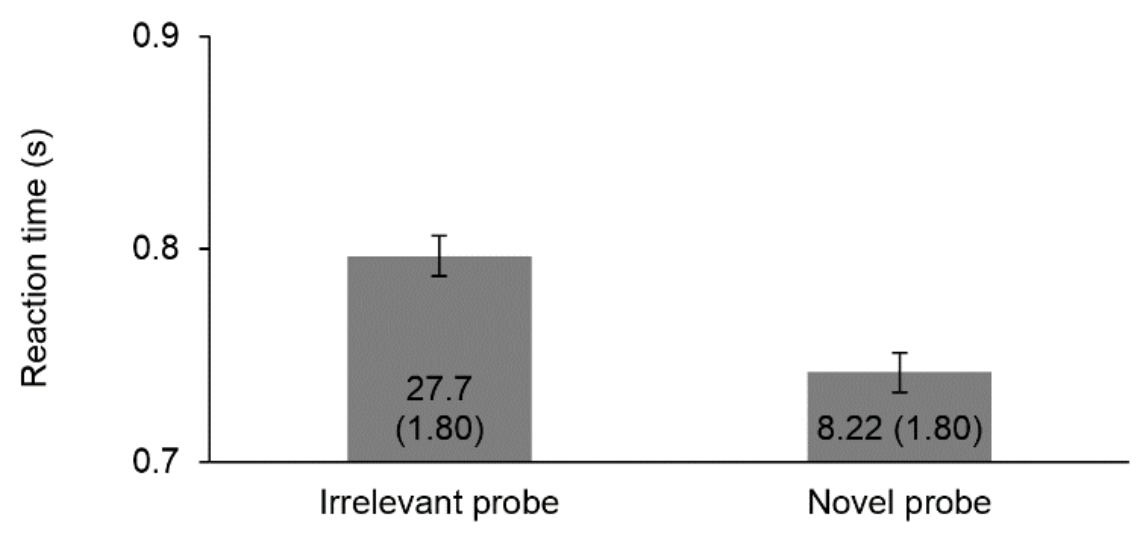

Figure 15. Reaction time data for two probe types for Experiment 4's working memory task. Participants exhibited an intrusion effect (longer reaction times to irrelevant probes than to novel probes). Numbers inside the bars indicate the error percentage for each condition.

Error rates are indicated using numerals in Figures 14 and 15. To assess speedaccuracy trade-offs, the same $2 \times 2$ ANOVA and $t$-test were run on error rates. The ANOVA revealed a statistically significant interaction, $F(1,24)=6.89, p=.015, \eta_{p}{ }^{2}=$ .223, a statistically significant main effect of relevant set size, $F(1,24)=104, p<.001$, $\eta_{\mathrm{p}}{ }^{2}=.812$, and a non-significant main effect of irrelevant set size, $F(1,24)=0.548, p=$ $.466, \mathrm{n}_{\mathrm{p}}{ }^{2}=.022, \mathrm{BF}_{01}=4.39$. Additionally, the paired-samples $t$-test revealed significantly more errors for the irrelevant probe condition than the novel probe condition, $t(24)=7.65, p<.001$. As with Experiment 3 , when participants made more errors, those conditions were associated with slower RTs, thus it is unlikely that the observed differences in RTs were due to speed/accuracy trade-offs.

Spatial blink task. Accuracy data for the spatial blink task are plotted in Figure 16. The 2 (distractor-target lag: 2 vs. 8) × 3 (distractor type: irrelevant vs. novel vs. target) within-subjects ANOVA yielded a significant main effect of distractor-target lag, $F(1,24)$ $=18.8, p<.001, \eta_{p}^{2}=.439$, a significant main effect of distractor type, $F(2,48)=16.0, p$ $<.001, \eta_{p}^{2}=.401$, and a significant interaction, $F(2,48)=17.0, p<.001, \eta_{p}{ }^{2}=.414$. 


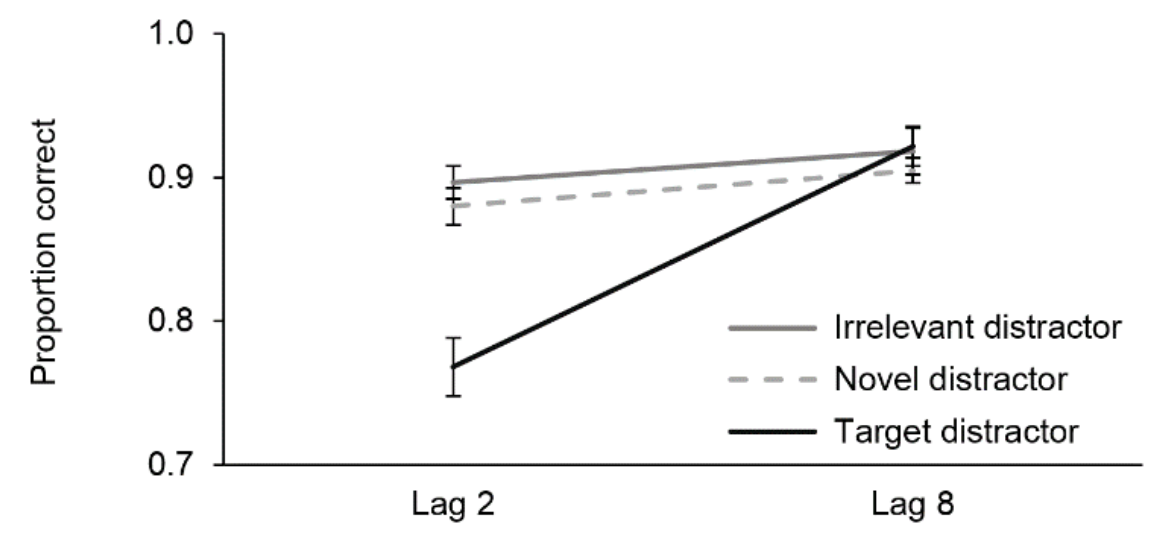

Figure 16. Accuracy data for the spatial blink (SB) task for Experiment 4, revealing contingent capture by target distractors, but not irrelevant distractors.

To assess for contingent capture, four planned paired-samples $t$-tests were conducted. Exhibiting a typical spatial blink, accuracy for the target distractor condition was significantly lower than accuracy for the novel distractor condition at lag $2, t(24)=4.82$, $p<.001, \mathrm{BF}_{10}=786$, one-tailed, but not at lag $8, t(24)=1.01, p=.319, \mathrm{BF}_{01}=2.99$. While the target distractor condition showed typical spatial blink results, the irrelevant distractor condition did not; accuracy did not significantly differ between the irrelevant distractor condition and the novel distractor condition at lag $2, t(24)=1.43, p=.917$, $\mathrm{BF}_{01}=10.5$, one-tailed, or at lag $8, t(24)=1.02, p=.319, \mathrm{BF}_{01}=2.98$. Though not significantly different, it is interesting to note that accuracy for the irrelevant distractor condition was higher than accuracy for the novel distractor condition during the spatial blink task, which would not be the case if the irrelevant distractor objects had formed an ACS and captured attention. Finally, accuracy was significantly higher for the irrelevant distractor condition than the target distractor condition at lag 2, $t(24)=5.89, p<0.001$, $\mathrm{BF}_{10}=4,556$, supporting the conclusion that participants did not adopt an ACS for the irrelevant objects. 


\section{Summary}

By incorporating target distractors in the spatial blink task, Experiment 4 provides a clear demonstration of the limited role ALTM plays in ACSs. Here, we observed that the target objects participants searched for in the spatial blink task formed an ACS and captured attention. However, the task irrelevant objects—which were represented in ALTM—did not. Thus, it would appear as if representing stimuli in ALTM is not sufficient for those same stimuli to form an ACS and capture visual attention in the way that searched-for objects do. Although we did not observe any evidence for contingent capture by task irrelevant objects, for completeness we again examined the relationship between individual differences in the intrusion effect and contingent capture; see Figure 17. The correlation was not statistically significant, $r=.384, p=.061, \mathrm{Cl}_{95}[-0.013$, $0.677], \mathrm{BF}_{10}=1.36$. Indeed, combining the correlation analyses across all four experiments, there was no evidence of a relationship between the intrusion effect and contingent capture, $r=-.136, p=.150, \mathrm{Cl}_{95}[-0.312,0.050], \mathrm{BF}_{01}=3.08$. These results suggest that a participant's ability to regulate attentional capture during the spatial blink task was unrelated to the extent to which they represented objects in ALTM. These results converge on the conclusion that ALTM is not the factor that determines which LTM representations will form an ACS, and which will not. 


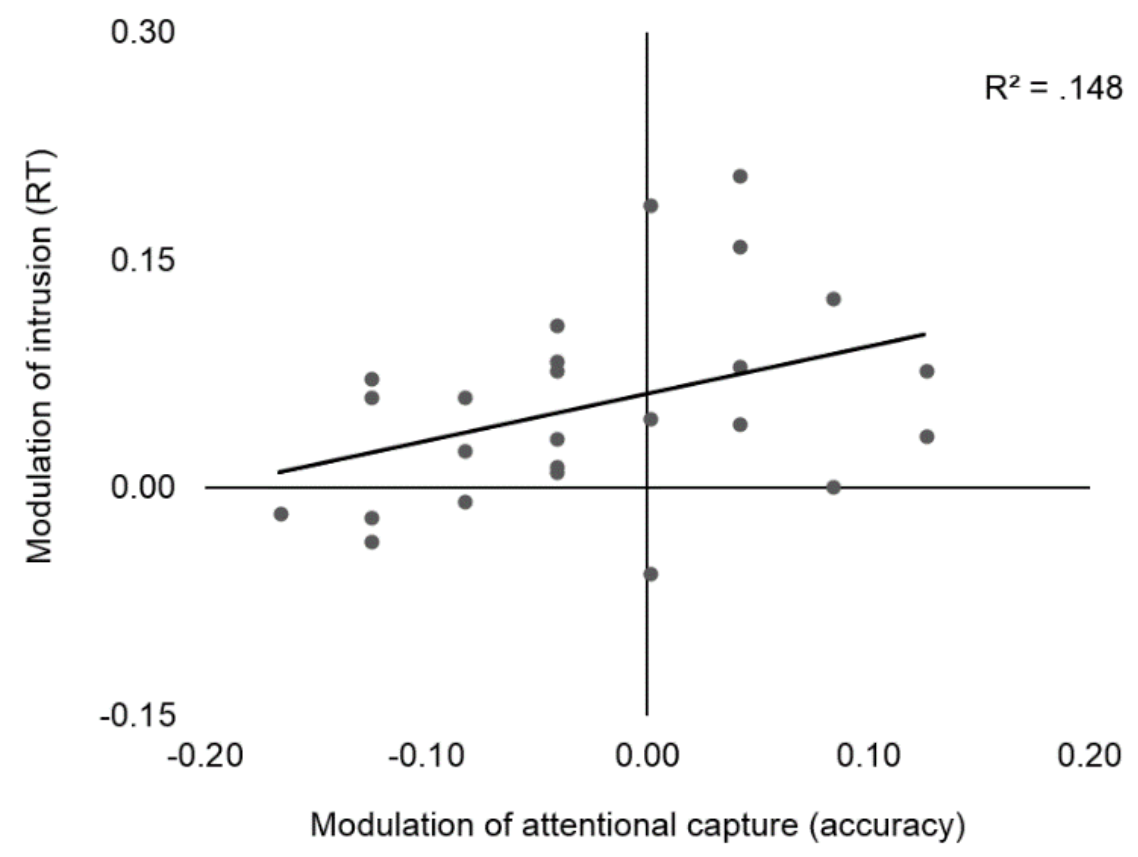

Figure 17. Correlation between individual differences in participants' ability to use an ACS to modulate attentional capture in the spatial blink task (novel distractor accuracy - irrelevant distractor accuracy), and to modulate the intrusion effect in the working memory task (irrelevant probe RT - novel probe RT) for Experiment 4. For both modulation measures, larger positive values indicate greater control.

\section{General Discussion}

In the present study, we investigated the ALTM account of LTM ACSs. In Experiments 1 and 2 we tested the prediction of this account that, when observers adopt an LTM ACS for a set of objects, those objects should be represented in ALTM, and produce intrusion effects in a working memory task. We found that the ACS objects did not exhibit a consistent intrusion effect. In Experiments 3 and 4, we induced participants to represent complex visual objects in ALTM and tested the prediction that those objects should be incorporated into participants' ACSs, and capture attention when presented as distractors in a spatial blink task. We found that those objects did not consistently capture attention. These results suggest that both tested predictions of the ALTM 
account are incorrect, and that representation in ALTM is not the factor that determines which LTM representations form an ACS.

\section{Is There Any Role for ALTM?}

Our results speak to both the necessity and sufficiency of representing objects in ALTM when adopting an ACS for those objects. Experiments 3 and 4 provide evidence that ALTM is not sufficient for adopting an ACS, as the irrelevant list objects were represented in ALTM but did not capture visual attention. If representation in ALTM was, by itself, sufficient for participants to adopt an ACS, then-by virtue of being represented in ALTM - the irrelevant list objects should have formed an ACS and captured attention during the spatial blink task. Also, the results from Experiments 1 and 2 suggest that ALTM may not be necessary for adopting an ACS, as the ACS objects captured attention during the spatial blink task, but these same objects did not always produce an intrusion effect during the working memory task. Perhaps the strongest evidence that representation in ALTM is not necessary for an object to be part of an LTM ACS, comes from the nonACS objects in Experiments 1 and 2. While it was not our intention, these objects captured attention (i.e., produced a spatial blink), suggesting that they were somewhat incorporated into participants ACSs, potentially due to being task relevant in the working memory task. Yet, these objects exhibited no evidence of intrusion effects; across both experiments, there was strong evidence favoring no difference in RTs between nonACS probes and novel probes during the working memory task, $\mathrm{BF}_{01}=18.6$ (Experiment $1, \mathrm{BF}_{01}=5.05$; Experiment 2, $\left.\mathrm{BF}_{01}=3.68\right)$. Without a suitable novel-distractor baseline for properly assessing capture by nonACS objects in Experiments 1 and 2, our assertion that ALTM is not necessary for LTM ACSs would benefit from further study. Regardless, the experiments presented here demonstrate that ALTM is not sufficient to support attentional capture by LTM ACSs, and, moreover, that factors other than representation in ALTM determine whether an LTM representation will bias attentional capture. 


\section{Attentional Control Settings vs. Attentional Templates}

The present ACS findings have potential implications for a closely related research topic: the attentional template. Attentional control settings and attentional templates share many core concepts. In particular, both describe how, when observers adopt a goal of searching their environment for a type of visual information, that goal shapes how attention is drawn to aspects of the environment (Bundesen, 1990; Duncan \& Humphrey, 1989; Folk et al., 1992, 1994). These two research areas, however, tend to diverge in how they are studied. Whereas most ACS research employs cueing tasks and other tasks thought to measure spatial attention, such as the spatial blink, attentional template research typically measures distractor costs during visual search; this divergence in how these phenomena are studied can lead to differing conclusions (Al-Aidroos, Harrison, \& Pratt, 2010; Folk \& Remington, 1998). Despite these differences, the present results may contribute to an ongoing debate in attentional template research regarding which memory systems store the attentional template, and when.

While there are many demonstrations suggesting that attentional templates can be stored in working memory (Olivers et al., 2011; Soto, Hodsoll, Rotshtein, \& Humphreys, 2008), some researchers have argued that templates are more commonly stored in LTM (Carlisle et al., 2011; Woodman et al., 2013). In particular, working memory may only store the identity of a search target that changes frequently (e.g., from trial to trial in an experiment); when the identity of the target remains constant over time, it can be offloaded to LTM, as evidenced by the absence of working memory-related electrophysiological activity (Carlisle et al., 2011). Conversely, recent evidence has emerged questioning the generalizability of this finding. In particular, when participants search for either of two targets, the attentional template continues to be stored in working memory, even when the identities of the targets are constant (Berggren \& Eimer, 2018). While this finding would seem to suggest that more complex templates are always stored in working memory, the present findings clarify that there are 
situations where complex templates (i.e., for 15 to 30 visual objects) are maintained in LTM.

\section{What Distinguishes ACS Representations in LTM, if not ALTM?}

If representation in ALTM is not the mechanism through which LTM ACSs are accomplished, then what is special about the LTM representations that comprise an ACS, such that these representations bias attentional capture, when other LTM representations do not? While there is no answer to this question in the literature thus far, one possibility is that the status of an object as part of an ACS is stored directly within the episodic memory trace associated with that object (i.e., stored as memory content, rather than through memory state). Upon encountering a visual object, memory traces associated with that object may be partially retrieved, including any information about whether a shift in attention is warranted. Given the traditional characterization of episodic LTM retrieval as a slow and effortful process (Yonelinas, 2002), it may seem surprising that episodic memories could be retrieved quickly and efficiently enough to support this role. More recently, however, it has been argued that episodic retrieval follows a two-stage process where, in the first stage, external stimuli (and internal representations) can trigger the rapid, effortless retrieval of memory traces (Degonda et al., 2005; Guild et al., 2014; Hannula, Tranel, \& Cohen, 2006; Moscovitch, 2008). Accordingly, the factor that distinguishes those LTM representations that comprise and ACS from those that do not may be stored within the contents of the memories themselves.

\section{Hybrid Visual and Memory Search}

The present findings also have implications for models of hybrid visual and memory search (Shiffrin \& Schneider, 1977; Wolfe, 2012). Similar to the present experiments, participants in hybrid search studies typically memorize a large set of everyday visual 
objects and then search their visual field for any one of those objects. In contrast to the present experiments, however, the search target in hybrid searches is presented concurrently with numerous visual distractors, and the memory and visual set sizes are manipulated (Cunningham \& Wolfe, 2014; Drew, Boettcher, \& Wolfe, 2016, 2017; Folk et al., 1994; Guild et al., 2014; Wolfe, 2012; Wolfe, Boettcher, Josephs, Cunningham, \& Drew, 2015). Thus, these studies examine how observers coordinate searching though their environment with searching through their memory, to find the visual stimulus that matches a target in memory. The main finding from these studies is that search RTs increase linearly with increasing numbers of visual distractors, and logarithmically with increasing numbers of targets in memory (Wolfe, 2012). Of minor interest, this research area adopted the term ALTM to describe the memory region where search targets are stored; though with some reservations (Cunningham \& Wolfe, 2014, p. 3). The present findings suggest a new label may be warranted.

Cunningham and Wolfe (2014) proposed a three-stage model to describe the coordination of visual attention and LTM during hybrid search, which has since been elaborated. Search stimuli are attended serially (Drew et al., 2017) based on their lowlevel featural properties, with each selected item being submitted to a massively parallel categorization and identification process, represented in visual working memory (Drew et al., 2016), and compared against the list of target objects stored in episodic (Guild et al., 2014) LTM. Notably, there are no direct interactions between visual attention and LTM in this model: Attention is only guided by low-level featural properties, not whole objects, and no comparison is made against the representations of the target objects in LTM until well after visual items are selected by attention. The lack of an interaction between attention and LTM is, however, inconsistent with the present findings, and those of Giammarco et al. (2016). Specifically, we observed that attention is guided by (i.e., capture is contingent on) the identities of the target objects, and thus LTM retrieval must have occurred early during processing. We suspect the difference between these two research areas relates to the two stages of episodic retrieval discussed above (Moscovitch, 2008). Whereas set size effects in hybrid search may be determined by a 
process that relies on the slow, conscious stage of retrieval, cueing effects and spatial blink costs may be more sensitive to attentional biases driven by rapid, unconscious recollection. As such, LTM ACS representations may be associated with both early and late effects during search.

\section{Conclusions}

The results from Experiments 1 and 2 presented here add to the growing literature demonstrating that LTM can interact with visual attention. Participants memorized and searched for 30 (Experiment 1) or 15 (Experiment 2) complex visual objects, and-due to the capacity limitations of working memory -it is clear that these objects were represented in LTM; yet these objects captured attention when presented as distractors during a spatial blink task. To better understand the processes supporting LTM ACSs, we investigated the ALTM account that LTM ACSs are accomplished by representing target objects in ALTM; however, the present findings do not support this account. The objects that comprise an LTM ACS are only inconsistently represented in ALTM (Experiments 1 and 2), and representing objects in ALTM is not sufficient for inducing an LTM ACS (Experiments 3 and 4). These results suggest that a factor other than ALTM determines which LTM representations form an ACS, and which do not.

\section{Overarching Discussion}

\section{Limitations and Future Directions}

While we as researchers strive to conduct the most rigorous, well-designed studies possible, research does not always go as planned.

The nonACS distractor objects in Experiments 1 and 2 captured attention during the spatial blink task. This was not what we expected, as previous research demonstrates that only ACS-matching stimuli should capture attention at short lags (Folk et al., 2002; 
Giammarco et al., 2016). It is possible that these objects captured attention as they were task relevant for the working memory portion of these experiments; participants had to respond to these objects when they appeared as memory probes. Thus, a useful future direction would be to include a novel distractor condition during the spatial blink task in order to replicate previous research and to provide a baseline measure for a distractor that does not capture attention.

Oberauer (2001) demonstrated that irrelevant list items were no longer represented in working memory within 1,000 ms after the offset of the cue, and that these items were represented in ALTM up to 5,000 ms (the longest cue-probe interval used). For Experiments 3 and 4, we used a cue-probe interval of $2,400 \mathrm{~ms}$, and demonstrated that, on working memory trials, the irrelevant list objects were represented in ALTM. However, due to the methodological changes made for these experiments-namely, that a spatial blink stream appeared instead of a memory probe-it is possible that something unexpected happened with the objects represented in ALTM. Perhaps the irrelevant list objects were no longer represented in ALTM by the time these objects appeared as distractors during the spatial blink task. Though this is unlikely to be the case, directly testing this possibility might be warranted. This could be done by having a spatial blink task after the offset of the probe for all trials, and to split into the different tasks when the distractor screen is supposed to appear. That is, to measure for the intrusion effect at the same point in the trial as testing for the presence of an ACS.

One last limitation is that, for Experiments 1 and 2, the working memory task was not the same as what was used by Oberauer (2001). It is possible that the intrusion effect found in Experiment 1, and the trending intrusion effect found in Experiment 2, were the result of some process unrelated to representation in ALTM. In order to strengthen the conclusion that ALTM is not necessary for adopting an LTM ACS, these two experiments could be re-done with a working memory task more similar as to what was used in Experiments 3 and 4. 


\section{Final Thoughts}

Here, we demonstrate that ALTM is not sufficient, and potentially not necessary, for adopting an LTM ACS. Yet we know that LTM ACSs still have the capacity to bias attentional capture, so how is this accomplished? This line of research represents the possibility to discover the mechanism through which LTM ACSs bias attentional capture. Importantly, this line of research is also an opportunity to explore the interaction between LTM and attention, and is an opportunity to bridge fields of research that have long been treated as separate.

\section{Disclosure statement}

The authors report no known conflict of interest.

\section{Funding}

This research was supported in part by an NSERC Canada Graduate Scholarship and a Queen Elizabeth II Graduate Scholarship in Science and Technology to Lindsay Plater, and an NSERC Discovery Grant (418507-201) and a CFI Grant (30374) to Dr. Naseem Al-Aidroos. 


\section{References}

Al-Aidroos, N., Harrison, S., \& Pratt, J. (2010). Attentional control settings prevent abrupt onsets from capturing visual spatial attention. Quarterly Journal of Experimental Psychology, 63(1), 31-41.

http://doi.org/10.1080/17470210903150738

Beck, V. M., Hollingworth, A., \& Luck, S. J. (2012). Simultaneous control of attention by multiple working memory representations. Psychological Science, 23(8), 887-898. http://doi.org/10.1177/0956797612439068

Berggren, N., \& Eimer, M. (2018). Visual working memory load disrupts template-guided attentional selection during visual search. British Journal of Psychology. http://doi.org/10.1111/bjop.12323

Brady, T. F., Konkle, T., Alvarez, G. A., \& Oliva, A. (2008). Visual long-term memory has a massive storage capacity for object details. Proceedings of the National Academy of Sciences of the United States of America, 105(38), 14325-14329. http://doi.org/10.1073/pnas.0803390105

Bundesen, C. (1990). A theory of visual attention. Psychological Review, 97(4), 523-47. http://doi.org/10.1037//0033-295X.97.4.523

Carlisle, N. B., Arita, J. T., Pardo, D., \& Woodman, G. F. (2011). Attentional templates in visual working memory. Journal of Neuroscience, 31(25), 9315-9322. http://doi.org/10.1523/JNEUROSCl.1097-11.2011

Cousineau, D. (2005). Confidence intervals in within-subject designs: A simpler solution to Loftus and Masson's method. Tutorials in Quantitative Methods for Psychology, 1(1), 42-45. http://doi.org/10.20982/tqmp.01.1.p042

Cowan, N. (1988). Evolving conceptions of memory storage, selective attention, and their mutual constraints within the human information-processing system. Psychological Bulletin, 104(2), 163-191. http://doi.org/10.1037/00332909.104.2.163

Cowan, N. (1999). An embedded-processes model of working memory. In A. Miyake \& P. Shah (Eds.), Models of working memory: Mechanisms of active maintenance 
and executive control (pp. 62-101). New York: Cambridge University Press. http://doi.org/10.1017/CBO9781139174909.006

Cunningham, C. A., \& Wolfe, J. M. (2014). The role of object categories in hybrid visual and memory search. Journal of Experimental Psychology: General, 143(4), 158599. http://doi.org/10.1037/a0036313

Degonda, N., Mondadori, C. R. A., Bosshardt, S., Schmidt, C. F., Boesiger, P., Nitsch, R. M., ... Henke, K. (2005). Implicit associative learning engages the hippocampus and interacts with explicit associative learning. Neuron, 46(3), 505-520.

http://doi.org/10.1016/j.neuron.2005.02.030

Drew, T., Boettcher, S. E. P., \& Wolfe, J. M. (2016). Searching while loaded: Visual working memory does not interfere with hybrid search efficiency but hybrid search uses working memory capacity. Psychonomic Bulletin and Review, 23(1), 201-212. http://doi.org/10.3758/s13423-015-0874-8

Drew, T., Boettcher, S. E. P., \& Wolfe, J. M. (2017). One visual search, many memory searches: An eye-tracking investigation of hybrid search. Journal of Vision, 17(11), 5. http://doi.org/10.1167/17.11.5

Duncan, J., \& Humphrey, G. W. (1989). Visual serach and stimulus similarity. Psychological Review, 96(3), 433-458. http://doi.org/10.1037/0033-295X.96.3.433 Folk, C. L., Leber, A. B., \& Egeth, H. E. (2002). Made you blink! Contingent attentional capture produces a spatial blink. Perception \& Psychophysics, 64(5), 741-753. http://doi.org/10.3758/BF03194741

Folk, C. L., \& Remington, R. (1998). Selectivity in distraction by irrelevant featural singletons: Evidence for two forms of attentional capture. Journal of Experimental Psychology: Human Perception and Performance, 24(3), 847-858. http://doi.org/10.1037/0096-1523.24.3.847

Folk, C. L., Remington, R. W., \& Johnston, J. C. (1992). Involuntary covert orienting is contingent on attentional control settings. Journal of Experimental Psychology: Human Perception and Performance, 18(4), 1030-1044. http://doi.org/10.1037/0096-1523.18.4.1030

Folk, C. L., Remington, R. W., \& Wright, J. H. (1994). The structure of attentional 
control: Contingent attentional capture by apparent motion, abrupt onset, and color. Journal of Experimental Psychology: Human Perception and Performance, 20(2), 317-329. http://doi.org/10.1037/0096-1523.20.2.317

Giammarco, M., Paoletti, A., Guild, E. B., \& Al-Aidroos, N. (2016). Attentional capture by items that match episodic long-term memory representations. Visual Cognition, 24(1), 78-101. http://doi.org/10.1080/13506285.2016.1195470

Goodhew, S. C., Kendall, W., Ferber, S., \& Pratt, J. (2014). Setting semantics:

Conceptual set can determine the physical properties that capture attention. Attention, Perception, and Psychophysics, 76(6), 1577-1589. http://doi.org/10.3758/s13414-014-0686-3

Guild, E. B., Cripps, J. M., Anderson, N. D., \& Al-Aidroos, N. (2014). Recollection can support hybrid visual memory search. Psychonomic Bulletin \& Review, 21(1), 142148. http://doi.org/10.3758/s13423-013-0483-3

Hannula, D. E., Tranel, D., \& Cohen, N. J. (2006). The long and the short of it: Relational memory impairments in amnesia, even at short lags. Journal of Neuroscience, 26(32), 8352-8359. http://doi.org/10.1523/JNEUROSCI.522205.2006

JASP-Team. (2017). JASP [Computer Software]. Retrieved from https://jasp-stats.org Luck, S. J., \& Vogel, E. (1997). The capacity of visual working memory for features and conjunctions. Nature, 390(6657), 279-281. http://doi.org/10.1038/36846

Morey, R. D. (2008). Confidence intervals from normalized data: A correction to Cousineau (2005). Tutorials in Quantitative Methods for Psychology, 4(2), 61-64. http://doi.org/10.20982/tqmp.04.2.p061

Morey, R. D., \& Rouder, J. N. (2011). Bayes Factor Approaches for Testing Interval Null Hypotheses. Psychological Methods, 16(4), 406-419. http://doi.org/10.1037/a0024377

Moscovitch, M. (2008). The hippocampus as a "stupid," domain-specific module: Implications for theories of recent and remote memory, and of imagination. Canadian Journal of Experimental Psychology, 62(1), 62-79. http://doi.org/10.1037/1196-1961.62.1.62 
Oberauer, K. (2001). Removing irrelevant information from working memory: A cognitive aging study with the modified Sternberg task. Journal of Experimental Psychology: Learning, Memory, and Cognition, 27(4), 948-957. http://doi.org/10.1037/02787393.27.4.948

Oberauer, K. (2002). Access to information in working memory: Exploring the focus of attention. Journal of Experimental Psychology: Learning, Memory, and Cognition, 28(3), 411-421. http://doi.org/10.1037//0278-7393.28.3.411

Oberauer, K., \& Hein, L. (2012). Attention to information in working memory. Current Directions in Psychological Science, 21(3), 164-169. http://doi.org/10.1177/0963721412444727

Olivers, C. N. L. (2009). What drives memory-driven attentional capture? The effects of memory type, display type, and search Type. Journal of Experimental Psychology: Human Perception and Performance, 35(5), 1275-1291. http://doi.org/10.1037/a0013896

Olivers, C. N. L., Meijer, F., \& Theeuwes, J. (2006). Feature-based memory-driven attentional capture: Visual working memory content affects visual attention. Journal of Experimental Psychology: Human Perception and Performance, 32(5), 12431265. http://doi.org/10.1037/0096-1523.32.5.1243

Olivers, C. N. L., Peters, J., Houtkamp, R., \& Roelfsema, P. R. (2011). Different states in visual working memory: When it guides attention and when it does not. Trends in Cognitive Sciences, 15(7), 327-334. http://doi.org/10.1016/j.tics.2011.05.004

Phillips, W. A. (1974). On the distinction between sensory storage and short-term visual memory. Perception \& Psychophysics, 16(2), 283-290. http://doi.org/10.3758/BF03203943

Posner, M. I., \& Cohen, Y. (1984). Components of visual orienting. Attention and Performance, 32, 531-556. http://doi.org/10.1162/jocn.1991.3.4.335

Shiffrin, R. M., \& Schneider, W. (1977). Controlled and automatic human information processing: II. Perceptual learning, automatic attending and a general theory. Psychological Review, 84(2), 127-190. http://doi.org/10.1037/0033-295X.84.2.127 Soto, D., Heinke, D., Humphreys, G. W., \& Blanco, M. J. (2005). Early, involuntary top- 
down guidance of attention from working memory. Journal of Experimental Psychology: Human Perception and Performance, 31(2), 248-261.

http://doi.org/10.1037/0096-1523.31.2.248

Soto, D., Hodsoll, J., Rotshtein, P., \& Humphreys, G. W. (2008). Automatic guidance of attention from working memory. Trends in Cognitive Sciences, 12(9), 342-348. http://doi.org/10.1016/j.tics.2008.05.007

Sternberg, S. (1966). High-speed scanning in human memory. Science, 153(3736), 652-654. http://doi.org/10.1126/science.153.3736.652

Wolfe, J. M. (2012). Saved by a log: How do humans perform hybrid visual and memory search? Psychological Science, 23(7), 698-703.

http://doi.org/10.1177/0956797612443968

Wolfe, J. M., Boettcher, S. E. P., Josephs, E. L., Cunningham, C. A., \& Drew, T. (2015). You look familiar, but I don't care: Lure rejection in hybrid visual and memory search is not based on familiarity. Journal of Experimental Psychology: Human Perception and Performance, 41(6), 1576-1587. http://doi.org/10.1037/xhp0000096

Woodman, G. F., Carlisle, N. B., \& Reinhart, R. M. G. (2013). Where do we store the memory representations that guide attention? Journal of Vision, 13(3), 1-17. http://doi.org/10.1167/13.3.1

Wyble, B., Folk, C., \& Potter, M. C. (2013). Contingent attentional capture by conceptually relevant images. Journal of Experimental Psychology: Human Perception and Performance, 39(3), 861-871. http://doi.org/10.1037/a0030517

Yonelinas, A. P. (2002). The nature of recollection and familiarity: A review of 30 years of research. Journal of Memory and Language, 46, 441-517. http://doi.org/10.1006/jmla.2002.2864 